\title{
Molecular scattering and Born-Oppenheimer approximation
}

\author{
Vania Sordoni \\ Dipartimento di Matematica \\ Università di Bologna \\ 40127 Bologna, Italia *
}

\begin{abstract}
In this paper, we study the scattering wave operators for a diatomic molecules by using the Born-Oppenheimer approximation. Assuming that the ratio $h^{2}$ between the electronic and nuclear masses is small, we construct adiabatic wave operators that, under some non trapping conditions, approximate the two-cluster wave operators up to any powers of the parameter $h$.
\end{abstract}

Keywords: Semiclassical analysis, Scattering theory, Born-Oppenheimer approximation, Pseudodifferential operators.

\section{Introduction}

In this paper, we discuss the scattering process of a diatomic molecule that dissociates into two ions. The complexity of the problem is due to the rather large number of particles, and, to overcome this difficulty, we apply the so called Born-Oppenheimer approximation. The idea of this method is based on the fact that, since the nuclei are much heavier than the electrons, their moving is slower and then the movement of the electrons, being almost instantaneous, is perceived by the nuclei as a surrounding electric field, if the parameter $h$, that represents the square root of the ratio between the electronic and nuclear masses, is small. Since the pioneering work of Born-Oppeneheimer (cfr.: [BoOp]) in 1927, many works are devoted to the investigation of Born-Oppenheimer approximation in spectral theory (cfr. : [CDS, Ha1, Ha2, Ha3, Ha4, Ha5, Ha6, HaJo, KMSW, Ma1, MaSo1, MaSo2, PST, SpTe, Te]), but only few (cfr. :[Ra, KMW1, KMW2, Je1, Je2, Je3, Je4]) investigate the applications of this method to scattering theory for molecules. In this papers, the authors provide a mathematical justification of the Born-Oppenheimer approximation for the two-cluster wave operators in

*Investigation supported by University of Bologna. Funds for selected research topics. 
diatomic molecular scattering. In particular, they construct an adiabatic wave operators that gives, under some non trapping assumptions, an approximation of the cluster wave operators modulo terms of order $\mathcal{O}(h)$, as the parameter $h$ tends to 0 . The purpose of this paper is to improve the results of [KMW1] by obtaining, in the case of smooth interactions, a good approximation of the cluster wave operators up to any power of $h$. Following the idea of [NeSo, So, MaSo1, MaSo2], we construct a quasi-invariant subspace and wave operators acting on such invariant subspace that approximates the original wave operators up to any order in $h$.

To begin with, let us introduce some notations.

Consider the Hamiltonian of a diatomic molecule with $N$ electrons:

$$
\begin{aligned}
P & =-h^{2} \Delta_{x_{1}}-h^{2} \Delta_{x_{2}}-\sum_{j=1}^{N} \Delta_{y_{j}}+\sum_{j=1}^{N}\left(W_{1, j}\left(y_{j}-x_{1}\right)+W_{2, j}\left(y_{j}-x_{2}\right)\right) \\
& +\sum_{\substack{i, j \in\{1, \ldots, N\} \\
i \neq j}} V_{i, j}\left(y_{i}-y_{j}\right)+W\left(x_{2}-x_{1}\right) .
\end{aligned}
$$

Here $x_{1}, x_{2} \in \mathbb{R}^{n}$ denote the positions of the two nuclei of mass $M, y_{j} \in \mathbb{R}^{n}, j=$ $1, . ., N$, the positions of the $N$ electrons with mass $m$ and $h:=\sqrt{\frac{m}{M}}$.

In the following we assume that $M$ is large enough with respect to $m$ so that $h$ can be regarded as a semiclassical parameter.

Moreover we assume that the potentials are smooth and short range.

Let $C=\left\{C_{1}, C_{2}\right\}$ be a cluster decomposition with $C_{j}=\{j\} \cup C_{j}^{\prime}, C_{j}^{\prime} \subset\{k, k=$ $1, . ., N\}, j=1,2$. The cluster Hamiltonian is given by,

$$
P_{c}=-h^{2} \Delta_{x_{1}}-h^{2} \Delta_{x_{2}}+Q_{0}(x)
$$

where,

$$
Q_{0}(x)=\sum_{k=1}^{2}\left(\sum_{j \in C_{k}^{\prime}}\left(-\Delta_{y_{j}}+W_{k, j}\left(y_{j}-x_{k}\right)\right)+\sum_{\substack{i, j \in C_{k}^{\prime} \\ i \neq j}} V_{i, j}\left(y_{i}-y_{j}\right)\right)
$$

and $P$ can be written as,

$$
P=-h^{2} \Delta_{x_{1}}-h^{2} \Delta_{x_{2}}+Q(x)=P_{c}+I_{c}(x)
$$

where

$$
Q(x)=Q_{0}(x)+I_{c}(x)
$$

and

$$
\begin{aligned}
I_{c}(x) & =W\left(x_{2}-x_{1}\right)+\sum_{j \in C_{1}^{\prime}} W_{2, j}\left(y_{j}-x_{2}\right)+\sum_{j \in C_{2}^{\prime}} W_{1, j}\left(y_{j}-x_{1}\right) \\
& +\sum_{i \in C_{1}^{\prime}, j \in C_{2}^{\prime}} V_{i, j}\left(y_{i}-y_{j}\right)+\sum_{i \in C_{2}^{\prime}, j \in C_{1}^{\prime}} V_{i, j}\left(y_{i}-y_{j}\right) .
\end{aligned}
$$


Let us fix $m$ eigenvalues $\hat{e}_{1}(h) \leq \hat{e}_{2}(h) \leq \ldots \leq \hat{e}_{m}(h)$, (dependent of $h$ ) of the cluster Hamiltonian $\hat{P}_{c}$ that one obtain from $P_{c}$ by fixing the center of mass and let us denote by $\hat{E}$ the spectral projection associated to these eigenvalues.

In the following, it will be shown that $\hat{e}_{j}(h)=e_{j}+\mathcal{O}\left(h^{2}\right)$ where $e_{j}$ are eigenvalues of $Q_{0}(x)$ (that are independent of $x$, since $Q_{0}(x)$ is translation invariant).

Let $f \in C_{0}^{\infty}(\mathbb{R})$ be some cutoff function.

In this paper we are interested in the Born-Oppenheimer approximation of the two cluster wave operators restricted to the the range of $\hat{E}$ i.e., denoting by $\hat{P}$ the operator that one obtain from $P$ by removing the kinetic energy of the total center of mass, we study the wave operators

$$
\Omega_{ \pm}^{c} \hat{E} f\left(\hat{P}_{c}\right)=s-\lim _{t \rightarrow \pm \infty} e^{i t \hat{P}} e^{-i t \hat{P}_{c}} \hat{E} f\left(\hat{P}_{c}\right)
$$

in $L^{2}\left(\mathbb{R}^{n(N+2)}\right)$.

Assuming that there there are exactly $m$ eigenvalues $\lambda_{j}\left(x_{2}-x_{1}\right), j=1, . ., m$, of $Q(x)$ that converge to $e_{j}$ when $\left|x_{2}-x_{1}\right| \rightarrow+\infty$, we will show that there exists an orthogonal projection $\hat{\Pi}$ that almost commutes with $P$ and that converges to $\hat{E}$ as $\left|x_{2}-x_{1}\right| \rightarrow+\infty$, such that, on the range of $\hat{E}$, the cluster wave operators $\Omega_{ \pm}^{c}$ can be decomposed into,

$$
\Omega_{ \pm}^{c} \hat{E} f\left(\hat{P}_{c}\right)=\Omega_{ \pm}^{N A D} \Omega_{ \pm}^{A D} f\left(\hat{P}_{c}\right)
$$

where

$$
\Omega_{ \pm}^{A D} f\left(\hat{P}_{c}\right)=s-\lim _{t \rightarrow \pm \infty} e^{i t \hat{\Pi} \hat{P} \hat{\Pi}} e^{-i t \hat{P}_{c}} \hat{E} f\left(\hat{P}_{c}\right)
$$

is the adiabatic wave operator and

$$
\Omega_{ \pm}^{N A D}=s-\lim _{t \rightarrow \pm \infty} e^{i t \hat{P}} e^{-i t \hat{\Pi} \hat{P} \hat{\Pi}} \hat{\Pi} f(\hat{\Pi} \hat{P} \hat{\Pi})
$$

is the non adiabatic wave operator. Moreover, if $\hat{E}$ is of rank one and a nontrapping condition on $\hat{\Pi} P \hat{\Pi}$ holds, one can prove that

$$
\Omega_{ \pm}^{N A D}=I+\mathcal{O}\left(h^{\infty}\right)
$$

and that $\Omega_{ \pm}^{A D}$ is unitary equivalent to the cluster wave operator of the heavy particles and the action of the electrons contributes as a small perturbation. Our results are formulate in a slightly different way with respect to the one of [Ra, KMW1, KMW2, Je1] since, here, we have preferred to keep the original coordinates instead of working in cluster atomic coordinates as in the articles mentioned above. The reason of the choice of this different coordinates system is related on the fact that, in cluster atomic coordinates, all the potentials become $h$-dependent and the construction of the projector $\hat{\Pi}$ is more difficult to obtain. On the other hand, it is easy to check that our results improve the one of [KMW1] by giving an approximation of the wave operators up to any power of the parameter $h$.

The plan of the paper is the following: 
In Section 2 we fix some notations and we state some preliminary results.

In Section 3, following the method of [NeSo, So, MaSo1, MaSo2], we construct two pseudodiffential operators with operator valued symbols, $\hat{\Pi}_{g}$ and $\hat{E}_{g}$ (depending on some cutoff function $g$ with Supp $f \subset\{g=1\})$, that are both projectors and almost commute with $\hat{P}$ and $\hat{P}_{c}$ respectively, modulo terms of order $\mathcal{O}\left(h^{\infty}\right)$. Moreover, if the kinetic energy remains bounded, $\hat{E}_{g}$ approximate $\hat{E}$, modulo error terms of order $\mathcal{O}\left(h^{\infty}\right)$.

In Section 4, we prove the existence of $\Omega_{ \pm}^{A D}$ and $\Omega_{ \pm}^{N A D}$ and the decomposition properties mentioned before.

In Section 5, assuming that the rank of $\hat{E}$ is one and that some non trapping conditions holds, we prove that the adiabatic wave operators are good approximation (modulo terms of order $\mathcal{O}\left(h^{\infty}\right)$ ) of the cluster wave operators restricted to the range of $\hat{E}$.

\section{Some preliminaries}

Let $C=\left\{C_{1}, C_{2}\right\}$ be, as before, a cluster decomposition with $C_{j}=\{j\} \cup C_{j}^{\prime}$, $C_{j}^{\prime} \subset\{k, k=1, \ldots, N\}$. Let us consider the cluster Hamiltonian,

$$
P_{c}=-h^{2} \Delta_{x_{1}}-h^{2} \Delta_{x_{2}}+Q_{0}(x)
$$

with $Q_{0}$ given by (1.2), and the molecular Hamiltonian,

$$
P=-h^{2} \Delta_{x_{1}}-h^{2} \Delta_{x_{2}}+Q(x)=P_{c}+I_{c}(x)
$$

with $Q(x)=Q_{0}(x)+I_{c}(x)$ and $I_{c}(x)$ given by (1.3).

In the following, we assume that all the potentials are smooth and short range, that is

(H1) $W, W_{1, j}, W_{2, j}$, and $V_{i, j}, i, j=1, \ldots, N, i \neq j$, are smooth and

$$
\left|\partial_{z}^{\alpha} W(z)\right|+\left|\partial_{z}^{\alpha} W_{1, j}(z)\right|+\left|\partial_{z}^{\alpha} W_{2, j}(z)\right|+\left|\partial_{z}^{\alpha} V_{i, j}(z)\right| \leq C\langle z\rangle^{-\delta-|\alpha|}
$$

for any $\alpha \in \mathbb{I N}^{n}$, for some $\delta>1$.

Let us remark that $P$ commutes with all the translations

$$
\begin{aligned}
& T_{k}: L^{2}\left(\mathbb{R}^{n(N+2)}\right) \rightarrow L^{2}\left(\mathbb{R}^{n(N+2)}\right), \\
& T_{k} u\left(x_{1}, x_{2}, y_{1}, . ., y_{N}\right)=u\left(x_{1}+k, x_{2}+k, y_{1}+k, . ., y_{N}+k\right), k \in \mathbb{R}^{n},
\end{aligned}
$$

and that $P_{c}$ commutes also with the translations

$$
\begin{aligned}
& T_{k_{1}, k_{2}}^{c}: L^{2}\left(\mathbb{R}^{n\left|C_{1}\right|}\right) \otimes L^{2}\left(\mathbb{R}^{n\left|C_{2}\right|}\right) \rightarrow L^{2}\left(\mathbb{R}^{n\left|C_{1}\right|}\right) \otimes L^{2}\left(\mathbb{R}^{n\left|C_{2}\right|}\right), \\
& T_{k_{1}, k_{2}}^{c}=T_{k_{1}}^{c} \otimes T_{k_{2}}^{c}, \\
& \left(T_{k_{i}}^{c} \phi\right)\left(x_{i},\left(y_{j}\right)_{j \in C_{i}^{\prime}}\right)=\phi\left(x_{i}+k_{i},\left(y_{j}+k_{i}\right)_{j \in C_{i}^{\prime}}\right), k_{i} \in \mathbb{R}^{n}, i=1,2 .
\end{aligned}
$$

Let us consider now, in each cluster, the change of variables $U=U_{1} \otimes U_{2}$ where $U_{i}, i=1,2$, is given by

$$
\begin{aligned}
R_{i} & :=\frac{x_{i}+h^{2} \sum_{j \in C_{i}^{\prime}} y_{j}}{1+h^{2}\left|C_{k}^{\prime}\right|} \\
\hat{y}_{j} & :=y_{j}-x_{i}, \quad j \in C_{i}^{\prime},
\end{aligned}
$$


for $i=1,2$, where $R_{i}$ represents the center of mass of the cluster $C_{i}$ and $\hat{y}_{j}$ the relative positions between the electrons and the nucleus in the cluster. Such a coordinate system defines a decomposition

$$
L^{2}\left(\mathbb{R}^{n(N+2)}\right)=L^{2}\left(\mathbb{R}^{2 n}\right) \otimes L^{2}\left(\mathbb{R}^{n N}\right)
$$

and we have

$$
U P_{c} U^{-1}=\left(-\alpha_{1}(h) \Delta_{R_{1}}-\alpha_{2}(h) \Delta_{R_{2}}\right) \otimes I+I \otimes \hat{Q}_{0}(h)
$$

where $\alpha_{i}(h)=\frac{h^{2}}{1+\left|C_{i}^{\prime}\right| h^{2}}$ and

$$
\hat{Q}_{0}(h)=\tilde{Q}_{0}+h^{2} \sum_{k=1}^{2}\left(\sum_{j \in C_{k}^{\prime}} D_{\hat{y}_{j}}\right)^{2}
$$

where

$$
\tilde{Q}_{0}=\sum_{k=1}^{2}\left(\sum_{j \in C_{k}^{\prime}}\left(-\Delta_{\hat{y}_{j}}+W_{k, j}\left(\hat{y}_{j}\right)\right)+\sum_{\substack{i, j \in C_{k}^{\prime} \\ i \neq j}} V_{i, j}\left(\hat{y}_{i}-\hat{y}_{j}\right)\right) .
$$

Assume that $\tilde{Q}_{0}$ has at least $m$ eigenvalues $e_{1} \leq e_{2} \leq \ldots \leq e_{m}$, repeated with their multiplicity, below its essential spectrum. By (2.3) and standard perturbation theory, it is easy to show that there are $m$ eigenvalues of $\hat{Q}_{0}(h)$, $\hat{e}_{1}(h) \leq \hat{e}_{2}(h) \leq \ldots \leq \hat{e}_{m}(h)$, such that

$$
\hat{e}_{j}(h)=e_{j}+\mathcal{O}\left(h^{2}\right) .
$$

Let $\hat{\pi}$ be the projection onto the eigenspace associated to

$$
\hat{\sigma}_{0}(h)=\left\{\hat{e}_{1}(h), \hat{e}_{2}(h), \ldots, \hat{e}_{m}(h)\right\} .
$$

In this paper we are interested in the Born-Oppenheimer approximation of the two cluster wave operators projected onto the eigenspace $\hat{\sigma}_{0}(h)$, i.e. to the study of,

$$
\Omega_{ \pm}^{c} \hat{E}=s-\lim _{t \rightarrow \pm \infty} e^{i t P} e^{-i t P_{c}} \hat{E}
$$

in $L^{2}\left(R^{n(N+2)}\right)$ where,

$$
\hat{E}=U^{-1}(I \otimes \hat{\pi}) U
$$

Since $P, P_{c}$ and $\hat{E}$ all commute with the translations (2.1) then $P, P_{c}$ and $\hat{E}$ also commute with the kinetic energy of the total center of mass of the system given by,

$$
K_{R}=\frac{h^{2}}{2+N h^{2}}\left(D_{x_{1}}+D_{x_{2}}+\sum_{j=1}^{N} D_{y_{j}}\right)^{2} .
$$

Hence, denoting by

$$
\hat{P}:=P-K_{R}, \quad \hat{P}_{c}:=P_{c}-K_{R}
$$


we have:

$$
\Omega_{ \pm}^{c}=s-\lim _{t \rightarrow \pm \infty} e^{i t P} e^{-i t P_{c}} \hat{E}=s-\lim _{t \rightarrow \pm \infty} e^{i t \hat{P}} e^{-i t \hat{P}_{c}} \hat{E}
$$

Moreover, denoting by $S_{x}$ the unitary operator on $L^{2}\left(\mathbb{R}^{n\left|C_{1}^{\prime}\right|}\right) \otimes L^{2}\left(\mathbb{R}^{n\left|C_{2}^{\prime}\right|}\right)$, given by,

$$
S_{x}:=S_{x_{1}} \otimes S_{x_{2}}, \quad\left(S_{x_{k}} \phi\right)(y)=\phi\left(\left(y_{i}+x_{k}\right)_{i \in C_{k}^{\prime}}\right), \quad k=1,2,
$$

we have

$$
Q_{0}(x)=S_{x} \tilde{Q}_{0} S_{-x}
$$

with $\tilde{Q}_{0}$ defined in (2.4), and

$$
Q(x)=S_{x} \tilde{Q}\left(x_{2}-x_{1}\right) S_{-x}
$$

where

$$
\begin{gathered}
\tilde{Q}\left(x_{2}-x_{1}\right)=\tilde{Q}_{0}+\tilde{I}_{c}\left(x_{2}-x_{1}\right) \\
\tilde{I}_{c}\left(x_{2}-x_{1}\right)=W\left(x_{2}-x_{1}\right)+\sum_{j \in C_{1}^{\prime}} W_{2, j}\left(y_{j}-\left(x_{2}-x_{1}\right)\right) \\
+\sum_{j \in C_{2}^{\prime}} W_{1, j}\left(y_{j}+\left(x_{2}-x_{1}\right)\right)+\sum_{i \in C_{1}^{\prime}, j \in C_{2}^{\prime}} V_{i, j}\left(y_{i}-y_{j}+\left(x_{2}-x_{1}\right)\right) .
\end{gathered}
$$

This shows that the spectrum of $Q_{0}(x)$ is independent of $x$ and that the one of $Q(x)$ depends on $x_{2}-x_{1}$ only.

Let $\Sigma\left(x_{2}-x_{1}\right)$ be the essential spectrum of $Q(x), \Sigma_{0}:=\liminf _{\left|x_{2}-x_{1}\right| \rightarrow+\infty} \Sigma\left(x_{2}-x_{1}\right)$ and let us assume that $e_{m}<\Sigma_{0}$. Then (see also Lemma 2.1 in [KMW1]), since

$$
\left|\tilde{I}_{c}\left(x_{2}-x_{1}\right)\right| \leq C\left\langle x_{2}-x_{1}\right\rangle^{-\delta}\langle y\rangle^{\delta}
$$

we have,

$$
\lim _{\left|x_{2}-x_{1}\right| \rightarrow+\infty} \tilde{Q}\left(x_{2}-x_{1}\right)=\tilde{Q}_{0}
$$

in the sense of strong resolvent convergence in $L^{2}\left(\mathbb{R}^{n N}\right)$. Hence, every point in the spectrum of $\tilde{Q}_{0}(x)$ is a limit of elements in $\operatorname{Sp}\left(\tilde{Q}\left(x_{2}-x_{1}\right)\right.$ ).

By using the decay of the eigenfunctions of $Q_{0}(x)$ associated to the eigenvalues $e_{j}$, we can show that there are at least $m$ eigenvalues of $Q(x)$ counted with their multiplicity that converges to some $e_{j}$ as $\left|x_{2}-x_{1}\right| \rightarrow+\infty$.

In the following we assume that they are stable, that is,

(H2) There are exactly $m$ eigenvalues $\lambda_{j}\left(x_{2}-x_{1}\right), j=1, . ., m$, (counted with their multiplicity) of $\tilde{Q}\left(x_{2}-x_{1}\right)$ (and then of $\left.Q(x)\right)$ such that, for any $j=1, . ., m$,

$$
\lim _{\left|x_{2}-x_{1}\right| \rightarrow+\infty} \lambda_{j}\left(x_{2}-x_{1}\right)=e_{j}
$$


and, setting $\sigma\left(x_{2}-x_{1}\right):=\left\{\lambda_{1}\left(x_{2}-x_{1}\right), \lambda_{2}\left(x_{2}-x_{1}\right), \ldots, \lambda_{m}\left(x_{2}-x_{1}\right)\right\}$, one has

$$
\left.\operatorname{dist}\left(\operatorname{Sp}\left(\tilde{Q}\left(x_{2}-x_{1}\right)\right) \backslash \sigma\left(x_{2}-x_{1}\right)\right), \sigma\left(x_{2}-x_{1}\right)\right) \geq c>0 .
$$

Now, let us take a complex oriented single loop $\Gamma\left(x_{2}-x_{1}\right)$ surrounding the set $\sigma\left(x_{2}-x_{1}\right)$ and leaving the rest of the spectrum of $\tilde{Q}\left(x_{2}-x_{1}\right)$ in its exterior. Without loss of generality one can suppose that $\Gamma\left(x_{2}-x_{1}\right)$ converges to $\Gamma_{0}$, a complex oriented single loop surrounding $\sigma_{0}:=\left\{e_{1}, e_{2}, \ldots, e_{m}\right\}$, and leaving the rest of the spectrum of $\tilde{Q}_{0}$ in its exterior.

The spectral projection of $Q(x)$ associated to $\sigma\left(x_{2}-x_{1}\right)$ can be written as

$$
\Pi_{0}(x)=\frac{1}{2 i \pi} \int_{\Gamma\left(x_{2}-x_{1}\right)}(z-Q(x))^{-1} d z .
$$

Under assumption (H2), one can also suppose that there exists $R>0$ sufficiently large such that

$$
\operatorname{Sp}(Q(x)) \cap \Gamma_{0}=\emptyset, \quad \text { for }\left|x_{1}-x_{2}\right|>R .
$$

Consequently, for $\left|x_{1}-x_{2}\right|>R$, we can write the spectral projection onto the eigenspaces associated to $\sigma_{0}$ and $\sigma\left(x_{2}-x_{1}\right)$ respectively as,

$$
E_{0}(x)=\frac{1}{2 i \pi} \int_{\Gamma_{0}}\left(z-Q_{0}(x)\right)^{-1} d z, \quad \Pi_{0}(x)=\frac{1}{2 i \pi} \int_{\Gamma_{0}}(z-Q(x))^{-1} d z .
$$

Our first result is the following (see also Theorem 2.2 in [KMW1]):

Proposition 2.1. Under the previous assumptions, one has, for any $\alpha \in \mathbb{N}^{6}$

$$
\left\|\partial_{x}^{\alpha}\left(\Pi_{0}(x)-E_{0}(x)\right)\right\|_{\mathcal{L}\left(L^{2}\left(\mathbb{R}^{n N}\right)\right)}=\mathcal{O}\left(\left\langle x_{2}-x_{1}\right\rangle^{-\delta}\right)
$$

Proof: We set, for $p \in \mathbb{R}$,

$$
\begin{aligned}
\zeta_{p}(x) & =\left\langle x_{2}-x_{1}\right\rangle^{-p}+\sum_{j \in C_{1}^{\prime}}\left\langle x_{2}-y_{j}\right\rangle^{-p}+\sum_{j \in C_{2}^{\prime}}\left\langle x_{1}-y_{j}\right\rangle^{-p}+\sum_{i \in C_{1}^{\prime}, j \in C_{2}^{\prime}}\left\langle y_{i}-y_{j}\right\rangle^{-p} \\
k_{p}(x) & =\sum_{j \in C_{1}^{\prime}}\left\langle y_{j}-x_{1}\right\rangle^{p}+\sum_{j \in C_{2}^{\prime}}\left\langle y_{j}-x_{2}\right\rangle^{p} .
\end{aligned}
$$

Then, for $\left|x_{1}-x_{2}\right|>R$, we have,

$$
\Pi_{0}(x)-E_{0}(x)=\frac{1}{2 i \pi} \int_{\Gamma_{0}}(z-Q(x))^{-1} I_{c}(x)\left(z-Q_{0}(x)\right)^{-1} d z,
$$

and, taking into account $(H 1)$ and applying standard commutator method, we get

$$
\left\|\zeta_{\delta}(x)^{-1}\left(\Pi_{0}(x)-E_{0}(x)\right)\right\|_{\mathcal{L}\left(L^{2}\left(\mathbb{R}^{n N}\right)\right)}=\mathcal{O}(1) .
$$


Let us remark that, thanks to the exponential decay of the eigenfunction of $\tilde{Q}_{0}$ or $\tilde{Q}\left(x_{2}-x_{1}\right)$ associated to $e_{j}$ and $\lambda_{j}\left(x_{2}-x_{1}\right), j=1, \ldots, m$, one has, for any $p>0$,

$$
\left\|k_{p}(x) E_{0}(x)\right\|_{\mathcal{L}\left(L^{2}\left(\mathbb{R}^{n N}\right)\right)}+\left\|k_{p}(x) \Pi_{0}(x)\right\|_{\mathcal{L}\left(L^{2}\left(\mathbb{R}^{n N}\right)\right)}=\mathcal{O}(1) .
$$

Moreover, since for any $p>0$ there exists a positive constant $C$ such that

$$
\zeta_{p}(x) \leq C\left\langle x_{2}-x_{1}\right\rangle^{-p} k_{p}(x)
$$

from (2.11) and (2.12) one get,

$$
\left\|\zeta_{p}(x) \Pi_{0}(x)\right\|_{\mathcal{L}\left(L^{2}\left(\mathbb{R}^{n N}\right)\right)}+\left\|\zeta_{p}(x) E_{0}(x)\right\|_{\mathcal{L}\left(L^{2}\left(\mathbb{R}^{n N}\right)\right)}=\mathcal{O}\left(\left\langle x_{2}-x_{1}\right\rangle^{-p}\right)
$$

for any $p>0$. Writing

$$
\Pi_{0}(x)-E_{0}(x)=\Pi_{0}(x)\left(\Pi_{0}(x)-E_{0}(x)\right)+\left(\Pi_{0}(x)-E_{0}(x)\right) E_{0}(x)
$$

and using (2.10) and (2.13) we obtain (2.9), for $\alpha=0$.

Suppose we have already proven the statement (2.9) for $|\alpha| \leq k$ and let us prove it for $|\alpha|=k+1$.

Using the identity (2.14), the inductive assumption and taking into account that $\partial_{x}^{\alpha} \Pi_{0}(x)$ and $\partial_{x}^{\alpha} E_{0}(x)$ are bounded operators on $L^{2}\left(\mathbb{R}^{n N}\right)$, one has, for $|\alpha|=k+1$,

$$
\begin{aligned}
\partial_{x}^{\alpha}\left(\Pi_{0}(x)-E_{0}(x)\right) & =\Pi(x) \partial_{x}^{\alpha}\left(\Pi_{0}(x)-E_{0}(x)\right) \\
& +\partial_{x}^{\alpha}\left(\Pi_{0}(x)-E_{0}(x)\right) E_{0}(x)+\mathcal{O}\left(\left\langle x_{2}-x_{1}\right\rangle^{-\delta}\right) .
\end{aligned}
$$

Then, since

$$
\partial_{x}^{\alpha}\left(\left(z-Q_{0}(x)\right)^{-1}\right), \quad \partial_{x}^{\alpha}\left((z-Q(x))^{-1}\right)
$$

are also bounded operators on $L^{2}\left(\mathbb{R}^{n N}\right)$ for $z \in \Gamma_{0}$, by assumption (H1), we obtain that

$$
\left\|\zeta_{\delta}(x)^{-1} \partial_{x}^{\alpha}\left(\Pi_{0}(x)-E_{0}(x)\right)\right\|_{\mathcal{L}\left(L^{2}\left(\mathbb{R}^{n N}\right)\right)}=\mathcal{O}(1)
$$

and then, using (2.15) and (2.13), we get (2.9) for $|\alpha|=k+1$.

\section{Construction of a Quasi-Invariant Subspace}

Let us construct here two orthogonal projections that almost commutes with the operator $\hat{P}$ and $\hat{P}_{c}$ defined in (2.6). We have the following:

Theorem 3.1. Assume (H1)-(H2). Then, for any $g \in C_{0}^{\infty}(\mathbb{R})$, there exist two orthogonal projections $\Pi_{g}, E_{g}$ on $L^{2}\left(\mathbb{R}^{(2+N) n}\right)$, that commute with the translations $T_{k}$ and $T_{k_{1}, k_{2}}^{c}$, defined in (2.1) and (2.2) respectively, and verify,

$$
\Pi_{g}=\Pi_{0}+\mathcal{O}(h), \quad E_{g}=E_{0}+\mathcal{O}(h) .
$$


Moreover, $\Pi_{g}$ and $E_{g}$ are $h$-admissible operators on $L^{2}\left(\mathbb{R}^{2 n} ; L^{2}\left(\mathbb{R}^{n N}\right)\right)$ uniformly bounded as operators from $L^{2}\left(\mathbb{R}^{2 n} ; L^{2}\left(\mathbb{R}^{n N}\right)\right)$ to $L^{2}\left(\mathbb{R}^{2 n} ; H^{2}\left(\mathbb{R}^{n N}\right)\right)$ and, for any $f \in C_{0}^{\infty}(\mathbb{R})$ with Supp $f \subset\{g=1\}$, and any $\ell \geq 0$, one has

$$
\hat{P}^{\ell}\left[f(\hat{P}), \Pi_{g}\right]=\mathcal{O}\left(h^{\infty}\right), \quad \hat{P}_{c}^{\ell}\left[f\left(\hat{P}_{c}\right), E_{g}\right]=\mathcal{O}\left(h^{\infty}\right)
$$

and,

$$
\begin{gathered}
\zeta_{s}^{-1}\left(\Pi_{g}-E_{g}\right) \zeta_{s^{\prime}}^{-1}=\mathcal{O}(1) \\
\zeta_{s}^{-1}\left(\hat{P}^{\ell}\left[f(\hat{P}), \Pi_{g}\right]-\hat{P}_{c}^{\ell}\left[f\left(\hat{P}_{c}\right), E_{g}\right]\right) \zeta_{s^{\prime}}^{-1}=\mathcal{O}(1)
\end{gathered}
$$

uniformly with respect to $h>0$, if $s+s^{\prime}=\delta$.

Proof: The key point in the proof of the theorem is (as in [NeSo, So, MaSo1, MaSo2]) the construction made in the following lemma:

Lemma 3.2. Under the previous assumptions, there exist functions

$$
\tilde{\pi}_{j}(x, \xi)=\sum_{|\alpha| \leq j} a_{\alpha, j}(x) \xi^{\alpha}, \quad \tilde{e}_{j}(x, \xi)=\sum_{|\alpha| \leq j} b_{\alpha, j}(x) \xi^{\alpha}
$$

polynomial of degree $j$ with respect to $\xi$, with smooth coefficients $a_{\alpha, j}(x), b_{\alpha, j}(x) \in$ $\mathcal{L}\left(L^{2}\left(\mathbb{R}^{n N}\right)\right)$, that commute with the translation $T_{k}$ and $T_{k_{1}, k_{2}}^{c}$ defined in (2.1) and (2.2) respectively, and such that the formal symbols

$$
\begin{aligned}
& \tilde{\pi}(x, \xi ; h)=\Pi_{0}(x)+\sum_{j \geq 1} \tilde{\pi}_{j}(x, \xi) h^{j} \\
& \tilde{e}(x, \xi ; h)=E_{0}(x)+\sum_{j \geq 1} \tilde{e}_{j}(x, \xi) h^{j}
\end{aligned}
$$

satisfy, at a formal series level,

$$
\begin{array}{cc}
\tilde{\pi}=\tilde{\pi}^{*}=\tilde{\pi} \# \tilde{\pi}, & \tilde{e}=\tilde{e}^{*}=\tilde{e} \# \tilde{e}, \\
\tilde{\pi} \# p-p \# \tilde{\pi}=0, & \tilde{e} \# p_{c}-p_{c} \# \tilde{e}=0, \\
\tilde{\pi} \# \hat{p}-\hat{p} \# \tilde{\pi}=0, & \tilde{e} \# \hat{p}_{c}-\hat{p}_{c} \# \tilde{e}=0 .
\end{array}
$$

and, in addition, one has, for any $\alpha \in \mathbb{I N}^{2 n}$ and for any $j \geq 1$,

$$
\left\|\zeta_{\delta}(x)^{-1} \partial_{x}^{\alpha}\left(a_{\alpha, j}(x)-b_{\alpha, j}(x)\right)\right\|_{\mathcal{L}\left(L^{2}\left(\mathbb{R}^{n N}\right)\right)}=\mathcal{O}(1) .
$$

Here, we have denoted by $p(x, \xi)=\xi_{1}^{2}+\xi_{2}^{2}+Q(x)$ the symbol of $P$ (resp.: $\hat{p}(x, \xi)$ the symbol of $\hat{P})$, by $p_{c}(x, \xi)=\xi_{1}^{2}+\xi_{2}^{2}+Q_{0}(x)$ the symbol of $P_{c}$ (resp.: $\hat{p}_{c}(x, \xi)$ the symbol of $\hat{P}_{c}$ ) and by \# the Weyl composition of symbols.

Proof: The construction of a symbol $\tilde{\pi}$ satisfying (3.5) and (3.6) can be done following the same arguments used in the proof of Lemma 3.1 of [So] (see also $[\mathrm{Sj} 2])$. 
Let us consider $\Gamma(x, \xi)=\left\{z \in \mathbb{C} ; z-\xi^{2} \in \Gamma\left(x_{2}-x_{1}\right)\right\}$, (resp.: $\Gamma_{0}(\xi)=\{z \in$ $\left.\left.\mathbb{C} ; z-\xi^{2} \in \Gamma_{0}\right\}\right)$.

For $z \in \Gamma(x, \xi)$ (resp.: $\left.z \in \Gamma_{0}(\xi)\right), p(x, \xi)-z\left(\right.$ resp.: $\left.p_{c}(x, \xi)-z\right)$ is invertible and $g_{0}(x, \xi ; z)=(p(x, \xi)-z)^{-1}\left(\operatorname{resp} .: g_{0}^{c}(x, \xi ; z)=\left(p_{c}(x, \xi)-z\right)^{-1}\right)$ is smooth and bounded.

Let us define the symbol $r(x, \xi ; h, z)\left(\operatorname{resp} .: r_{c}(x, \xi ; h, z)\right)$, as,

$$
\begin{array}{ll} 
& (p(x, \xi)-z) \# g_{0}(x, \xi ; z)=1-r(x, \xi ; h, z), \\
\left(\text { resp. : } \quad\left(p_{c}(x, \xi)-z\right) \# g_{0}^{c}(x, \xi ; z)=1-r_{c}(x, \xi ; h, z)\right)
\end{array}
$$

and the symbol $g(x, \xi ; h, z)=\sum_{j} h^{j} g_{j}(x, \xi ; h, z)$ (respectively, $g^{c}(x, \xi ; h, z)=$ $\left.\sum_{j} h^{j} g_{j}^{c}(x, \xi ; h, z)\right)$ as,

$$
g=g_{0}+g_{0} \# \sum_{j \geq 1} r^{\# j}, \quad\left(\text { resp. }: g^{c}=g_{0}^{c}+g_{0}^{c} \# \sum_{j \geq 1} r_{c}^{\# j}\right) .
$$

One can check that, for $j \geq 1, g_{j}(x, \xi ; h, z)$ (resp.: $\left.g_{j}^{c}(x, \xi ; h, z)\right)$ is given by (see e.g. [Ba])

$$
\begin{aligned}
& g_{j}=g_{0} \sum_{m=1}^{2 j} \sum_{\left(\left(\alpha_{i}\right),\left(\beta_{i}\right)\right) \in E_{m}^{j}} C_{m}\left((\alpha)_{i},(\beta)_{i}\right) \prod_{i=1}^{m}\left(\left(\partial_{x}^{\alpha_{i}} \partial_{\xi}^{\beta_{i}} p\right) g_{0}\right) \\
& g_{j}^{c}=g_{0}^{c} \sum_{m=1}^{2 j} \sum_{\left(\left(\alpha_{i}\right),\left(\beta_{i}\right)\right) \in E_{m}^{j}} C_{m}\left((\alpha)_{i},(\beta)_{i}\right) \prod_{i=1}^{m}\left(\left(\partial_{x}^{\alpha_{i}} \partial_{\xi}^{\beta_{i}} p_{c}\right) g_{0}^{c}\right)
\end{aligned}
$$

where $E_{m}^{j}=\left\{\left(\left(\alpha_{i}\right),\left(\beta_{i}\right)\right) \in\left(\mathbb{Z}_{+}^{2 n}\right)^{m} \times\left(\mathbb{Z}_{+}^{2 n}\right)^{m} ;\left|\sum_{i=1}^{m} \alpha_{i}\right|=\left|\sum_{i=1}^{m} \beta_{i}\right|=j\right\}$ and $C_{m}\left((\alpha)_{i},(\beta)_{i}\right)$ are universal constants. Let us define

$$
\begin{aligned}
& \tilde{\pi}(x, \xi ; h)=\Pi_{0}(x)+\sum_{j \geq 1} \tilde{\pi}_{j}(x, \xi) h^{j}, \\
& \tilde{e}(x, \xi ; h)=E_{0}(x)+\sum_{j \geq 1} \tilde{e}_{j}(x, \xi) h^{j},
\end{aligned}
$$

where

$$
\begin{aligned}
\tilde{\pi}_{j}(x, \xi) & =\frac{i}{2 \pi} \oint_{\Gamma(x, \xi)} g_{j}(x, \xi, z) d z, \\
\tilde{e}_{j}(x, \xi) & =\frac{i}{2 \pi} \oint_{\Gamma_{0}(\xi)} g_{j}^{c}(x, \xi, z) d z .
\end{aligned}
$$

Then $\tilde{\pi}, \tilde{e}$ satisfies (3.5) and (3.6) and, by making the change of variable $z \rightarrow$ 
$z-\xi^{2}$ in the integrals (3.11), (3.12), one can easily check that

$$
\begin{aligned}
\tilde{\pi}_{j}(x, \xi) & =\frac{i}{2 \pi} \oint_{\Gamma\left(x_{2}-x_{1}\right)} g_{j}\left(x, \xi, z+\xi^{2}\right) d z=\sum_{|\alpha| \leq j} a_{\alpha, j}(x) \xi^{\alpha}, \\
\tilde{e}_{j}(x, \xi) & =\frac{i}{2 \pi} \oint_{\Gamma_{0}} g_{j}^{c}\left(x, \xi, z+\xi^{2}\right) d z=\sum_{|\alpha| \leq j} b_{\alpha, j}(x) \xi^{\alpha}
\end{aligned}
$$

are polynomials operator-valued functions of degree $j$, with smooth coefficients. Let us show that the construction of $\tilde{\pi}(x, \xi ; h)$ is unique. If

$$
\hat{\pi}(x, \xi ; h)=\Pi_{0}(x)+\sum_{j \geq 1} \hat{\pi}_{j}(x, \xi) h^{j},
$$

is an other formal series with $\hat{\pi}_{j}(x, \xi)$ polynomials operator-valued functions of degree $j$, with smooth coefficients that satisfy (3.5), and (3.6), then

$$
\hat{\pi} \# g-g \# \hat{\pi}=0 .
$$

Hence, by construction of $\tilde{\Pi}$,

$$
\hat{\pi} \# \tilde{\pi}-\tilde{\pi} \# \hat{\pi}=0,
$$

and

$$
(1-\tilde{\pi}) \# \hat{\pi} \#(1-\tilde{\pi}) \# \hat{\pi}=(1-\tilde{\pi}) \# \hat{\pi} .
$$

Since, on the other hand, $(1-\tilde{\pi}) \# \hat{\pi}=\mathcal{O}(h)$ then $(1-\tilde{\pi}) \# \hat{\pi}=(1-\hat{\pi}) \# \tilde{\pi}=0$. Thus

$$
\hat{\pi}-\tilde{\pi}=(1-\tilde{\pi}) \# \hat{\pi}-(1-\hat{\pi}) \# \tilde{\pi}-\hat{\pi} \# \tilde{\pi}+\tilde{\pi} \# \hat{\pi}=0 .
$$

In the same way one can prove that $\tilde{e}(x, \xi ; h)$ is unique.

Let us define now

$$
\hat{\pi}(x, \xi ; h):=\Pi_{0}(x)+\sum_{j \geq 1} \hat{\pi}_{j}(x, \xi) h^{j}, \quad \hat{e}(x, \xi ; h):=E_{0}(x)+\sum_{j \geq 1} \hat{e}_{j}(x, \xi) h^{j}
$$

with

$$
\hat{\pi}_{j}(x, \xi):=\sum_{|\alpha| \leq j} T_{k} a_{\alpha, j}(x) T_{-k} \xi^{\alpha}, \quad \hat{e}_{j}(x, \xi):=\sum_{|\alpha| \leq j} T_{k_{1}, k_{2}}^{c} b_{\alpha, j}(x) T_{-k_{1},-k_{2}}^{c} \xi^{\alpha}
$$

Then, $\hat{\pi}$ and $\hat{e}$ satisfy (3.5) and (3.6) and then, by the uniqueness, one obtain that $\hat{\pi}_{j}(x, \xi)=\tilde{\pi}_{j}(x, \xi)$ and $\hat{e}_{j}(x, \xi)=\tilde{e}_{j}(x, \xi)$.

Hence $a_{\alpha, j}(x)$ and $b_{\alpha, j}(x)$ commutes with $T_{k}$ for any $k \in \mathbb{R}^{n}$ and this proves that (3.7) holds. By writing, for $\left|x_{2}-x_{2}\right|>R$ with $R$ sufficiently large,

$$
\tilde{\pi}_{j}(x, \xi)-e_{j}(x, \xi)=\frac{i}{2 \pi} \oint_{\Gamma_{0}}\left(g_{j}\left(x, \xi, z+\xi^{2}\right)-g_{j}^{c}\left(x, \xi, z+\xi^{2}\right)\right) d z
$$

and using (3.9), (3.10) one can easily prove the decay properties (3.8) as $\left|x_{2}-x_{1}\right| \rightarrow \infty$. 
End of the proof of Theorem 3.1: To end the proof of the theorem, let us follow the idea of [So] and [MaSo2].

Let $g \in C_{0}^{\infty}(\mathbb{R})$. Let us denote by $\tilde{\Pi}_{j}$ and $\tilde{E}_{j}$ the differential operator with (Weyl) symbol $\tilde{\pi}_{j}$ and $\tilde{e}_{j}$. Writing $g\left(\hat{P}_{c}\right) \tilde{E}_{k}=g\left(\hat{P}_{c}\right)\left(\hat{P}_{c}+i\right)^{N}\left(\hat{P}_{c}+i\right)^{-N} \tilde{E}_{k}$ and $g(\hat{P}) \tilde{\Pi}_{k}=g(\hat{P})(\hat{P}+i)^{N}(\hat{P}+i)^{-N} \tilde{\Pi}_{k}$, we see that the operators $g(\hat{P}) \tilde{\Pi}_{k}$, $g\left(\hat{P}_{c}\right) \tilde{E}_{k}(k \geq 0)$ are all $h$-admissible operators. In particular, they are all bounded, uniformly with respect to $h$ and it is easy to show that, by $(3.8)$, one has

$$
\left\|\zeta_{s}(x)^{-1}\left(g(\hat{P}) \tilde{\Pi}_{k}-\tilde{g}\left(\hat{P}_{c}\right) \tilde{E}_{k}\right) \zeta_{s^{\prime}}(x)^{-1}\right\|_{\mathcal{L}\left(L^{2}\left(\mathbb{R}^{n N}\right)\right)}=\mathcal{O}(1)
$$

if $s+s^{\prime}=\delta$. Moreover, one can resum in a standard way the formal series of operators $\sum_{k=0}^{\infty} h^{k} g\left(\hat{P}_{c}\right) \tilde{E}_{k}, \sum_{k=0}^{\infty} h^{k} g(P) \tilde{\Pi}_{k}$ (see, e.g., [Ma2] Lemma 2.3.3), in such a way that, if we denote by $E(g)$ and $\Pi(g)$ such resummations, (see also Lemma 6.2 in [MaSo2]) for any $\ell \geq 0$, one has,

$\left\|\hat{P}_{c}^{\ell}\left(E(g)-E(g)^{*}\right)\right\|_{\mathcal{L}\left(L^{2}\left(\mathbb{R}^{n(N+2)}\right)\right.}+\left\|\hat{P}^{\ell}\left(\Pi(g)-\Pi(g)^{*}\right)\right\|_{\mathcal{L}\left(L^{2}\left(\mathbb{R}^{n(N+2)}\right)\right.}=\mathcal{O}\left(h^{\infty}\right)$.

and,

$$
\left\|\zeta_{s}(x)^{-1}(\Pi(g)-E(g)) \zeta_{s^{\prime}}(x)^{-1}\right\|_{\mathcal{L}\left(L^{2}\left(\mathbb{R}^{n N}\right)\right)}=\mathcal{O}(1)
$$

if $s+s^{\prime}=\delta$. We set,

$$
\begin{gathered}
\left.\tilde{E}_{g}:=E(g)+E(g)^{*}-\frac{1}{2}\left(g\left(\hat{P}_{c}\right)\right) E(g)^{*}+E(g) g\left(\hat{P}_{c}\right)\right)+\left(1-g\left(\hat{P}_{c}\right)\right) E_{0}\left(1-g\left(\hat{P}_{c}\right)\right) \\
\left.\tilde{\Pi}_{g}:=\Pi(g)+\Pi(g)^{*}-\frac{1}{2}(g(\hat{P})) \Pi(g)^{*}+\Pi(g) g(\hat{P})\right)+(1-g(\hat{P})) \Pi_{0}(1-g(\hat{P})) .
\end{gathered}
$$

Then, $\tilde{E}_{g}, \tilde{\Pi}_{g}$ are selfadjoint $h$-admissible operator, and since $\Pi(g)=g(\hat{P}) \Pi_{0}+$ $\mathcal{O}(h), E(g)=g\left(\hat{P}_{c}\right) E_{0}+\mathcal{O}(h)$, we have,

$$
\begin{aligned}
& \left\|\tilde{\Pi}_{g}-\Pi_{0}\right\|_{\mathcal{L}\left(L^{2}\left(\mathbb{R}^{n(N+2)}\right)\right)}+\left\|\tilde{\Pi}_{g}^{2}-\tilde{\Pi}_{g}\right\|_{\mathcal{L}\left(L^{2}\left(\mathbb{R}^{n(N+2)}\right)\right)}=\mathcal{O}(h) \\
& \left\|\tilde{E}_{g}-E_{0}\right\|_{\mathcal{L}\left(L^{2}\left(\mathbb{R}^{n(N+2)}\right)\right)}+\left\|\tilde{E}_{g}^{2}-\tilde{E}_{g}\right\|_{\mathcal{L}\left(L^{2}\left(\mathbb{R}^{n(N+2)}\right)\right)}=\mathcal{O}(h) .
\end{aligned}
$$

Arguing as in [MaSo2], Theorem 6.1, we obtain that, for any $f \in C_{0}^{\infty}(\mathbb{R})$ such that $\operatorname{Supp} f \subset\{g=1\}$,

$$
\left\|\hat{P}_{c}^{\ell}\left[f\left(\hat{P}_{c}\right), \tilde{E}_{g}\right]\right\|_{\mathcal{L}\left(L^{2}\left(\mathbb{R}^{n(N+2)}\right)\right)}+\left\|\hat{P}^{\ell}\left[f(\hat{P}), \tilde{\Pi}_{g}\right]\right\|_{\mathcal{L}\left(L^{2}\left(\mathbb{R}^{n(N+2)}\right)\right)}=\mathcal{O}\left(h^{\infty}\right)
$$

and

$$
\hat{P}_{c}^{\ell}\left(\tilde{E}_{g}^{2}-\tilde{E}_{g}\right) f\left(\hat{P}_{c}\right)=\mathcal{O}\left(h^{\infty}\right), \quad \hat{P}^{\ell}\left(\hat{\Pi}_{g}^{2}-\tilde{\Pi}_{g}\right) f(\tilde{P})=\mathcal{O}\left(h^{\infty}\right)
$$

for any $\ell \geq 0$.

Moreover, one has

$$
\left\|\zeta_{s}(x)^{-1}\left(\tilde{\Pi}_{g}-\tilde{E}_{g}\right) \zeta_{s^{\prime}}(x)^{-1}\right\|_{\mathcal{L}\left(L^{2}\left(\mathbb{R}^{n N}\right)\right)}=\mathcal{O}(1)
$$


and one can easily check that

$$
\left\|\zeta_{s}(x)^{-1}\left(\hat{P}^{\ell}\left[f(\hat{P}), \tilde{\Pi}_{g}\right]-\hat{P}_{c}^{\ell}\left[f\left(\hat{P}_{c}\right), \tilde{E}_{g}\right]\right) \zeta_{s^{\prime}}(x)^{-1}\right\|_{\mathcal{L}\left(L^{2}\left(\mathbb{R}^{n N}\right)\right)}=\mathcal{O}(1) .
$$

if $s+s^{\prime}=\delta$. Then, following the arguments of [Ne1, Ne2, NeSo, So, MaSo1, MaSo2], for $h$ small enough, we can define the following orthogonal projections:

$$
E_{g}:=\frac{i}{2 \pi} \int_{|z-1|=\frac{1}{2}}\left(\tilde{E}_{g}-z\right)^{-1} d z, \quad \Pi_{g}:=\frac{i}{2 \pi} \int_{|z-1|=\frac{1}{2}}\left(\tilde{\Pi}_{g}-z\right)^{-1} d z .
$$

Using [So], Formula (3.9), and [Ne1], Proposition 3, we obtain from (3.17),

$$
\hat{P}_{c}^{\ell}\left(E_{g}-\tilde{E}_{g}\right) f\left(\hat{P}_{c}\right)=\mathcal{O}\left(h^{\infty}\right), \quad \hat{P}^{\ell}\left(\Pi_{g}-\tilde{\Pi}_{g}\right) f(\hat{P})=\mathcal{O}\left(h^{\infty}\right),
$$

and, using (3.15), (3.16) (3.18) and (3.19), we complete the proof of the Theorem.

Remark 3.3. Writing, for any $f \in C_{0}^{\infty}(\mathbb{R})$ with Supp $f \subset\{g=1\}$,

$$
\begin{aligned}
f(\hat{P})\left[\hat{P}, \Pi_{g}\right] & =\left[f(\hat{P}) \hat{P}, \Pi_{g}\right]-\left[f(\hat{P}), \Pi_{g}\right] \hat{P} \\
f\left(\hat{P}_{c}\right)\left[\hat{P}_{c}, E_{g}\right] & =\left[f\left(\hat{P}_{c}\right) \hat{P}_{c}, E_{g}\right]-\left[f\left(\hat{P}_{c}\right), E_{g}\right] \hat{P}_{c}
\end{aligned}
$$

and applying the results of Theorem 3.1, we also get

$$
f(\hat{P})\left[\hat{P}, \Pi_{g}\right]=\mathcal{O}\left(h^{\infty}\right), \quad f\left(\hat{P}_{c}\right)\left[\hat{P}_{c}, E_{g}\right]=\mathcal{O}\left(h^{\infty}\right)
$$

and,

$$
\zeta_{s}^{-1}\left(f(\hat{P})\left[\hat{P}, \Pi_{g}\right]-f\left(\hat{P}_{c}\right)\left[\hat{P}_{c}, E_{g}\right]\right) \zeta_{s^{\prime}}^{-1}=\mathcal{O}(1)
$$

uniformly with respect to $h>0$, if $s+s^{\prime}=\delta$.

Let us show now that $E_{g}$ is a good approximation of $\hat{E}$. We have the following:

Proposition 3.4. For any $g \in C_{0}^{\infty}(\mathbb{R})$ and for any $\tilde{g} \in C_{0}^{\infty}(\mathbb{R})$ with Supp $\tilde{g} \subset$ $\{g=1\}$, we have

$$
\left(E_{g}-\hat{E}\right) \tilde{g}\left(\hat{P}_{c}\right)=\mathcal{O}\left(h^{\infty}\right) .
$$

Proof: Since $\hat{E}$ is a spectral projection of $\hat{P}_{c}$, from (3.21) we also have

$$
\tilde{g}\left(\hat{P}_{c}\right)\left[\hat{E}, E_{g}\right]=\mathcal{O}\left(h^{\infty}\right)
$$

for any $\tilde{g} \in C_{0}^{\infty}(\mathbb{R})$ with $\operatorname{Supp} \tilde{g} \subset\{g=1\}$. Hence

$$
\left(1-E_{g}\right) \hat{E}\left(1-E_{g}\right) \hat{E} \tilde{g}\left(\hat{P}_{c}\right)=\left(1-E_{g}\right) \hat{E} \tilde{g}\left(\hat{P}_{c}\right)+\mathcal{O}\left(h^{\infty}\right)
$$

and, since $\left(1-E_{g}\right) \hat{E}=\mathcal{O}(h)$, we easily deduce,

$$
\left(1-E_{g}\right) \hat{E} \tilde{g}\left(\hat{P}_{c}\right)=\mathcal{O}\left(h^{\infty}\right) .
$$


By the same arguments one also obtains that

$$
E_{g}(1-\hat{E}) \tilde{g}\left(\hat{P}_{c}\right)=\mathcal{O}\left(h^{\infty}\right) .
$$

Thus

$$
\left(E_{g}-\hat{E}\right) \tilde{g}\left(\hat{P}_{c}\right)=E_{g}(1-\hat{E}) \tilde{g}\left(\hat{P}_{c}\right)-\left(1-E_{g}\right) \hat{E} \tilde{g}\left(\hat{P}_{c}\right)+\mathcal{O}\left(h^{\infty}\right)=\mathcal{O}\left(h^{\infty}\right) .
$$

Remark 3.5. By using Theorem 3.1 and Proposition 3.4 one easily obtains that, for any $N \in \mathbb{N}$, there exist $e_{k, j} \in \mathbb{R}, j=2, \ldots, N$, and $C_{N}>0$ such that,

$$
\left|\hat{e}_{k}(h)-e_{k}-\sum_{j=2}^{N} e_{k, j} h^{j}\right| \leq C_{N} h^{N+1}
$$

for $k=1, \ldots, m$.

Theorem 3.6. Under assumption (H1)-(H2), for any $g \in C_{0}^{\infty}(\mathbb{R})$, there exists an orthogonal projection $\hat{\Pi}_{g}$ on $L^{2}\left(\mathbb{R}^{(2+N) n}\right)$, that commute with the translations $T_{k}$, defined in (2.1), and verifies,

$$
\hat{\Pi}_{g}=\Pi_{0}+\mathcal{O}(h) .
$$

Moreover, $\hat{\Pi}_{g}$ is an $h$-admissible operator on $L^{2}\left(\mathbb{R}^{2 n} ; L^{2}\left(\mathbb{R}^{n N}\right)\right)$ uniformly bounded as operators from $L^{2}\left(\mathbb{R}^{2 n} ; L^{2}\left(\mathbb{R}^{n N}\right)\right)$ to $L^{2}\left(\mathbb{R}^{2 n} ; H^{2}\left(\mathbb{R}^{n N}\right)\right)$ and for any $f \in C_{0}^{\infty}(\mathbb{R})$ with Supp $f \subset\{g=1\}$, and one has

$$
\zeta_{s}^{-1}\left(\hat{\Pi}_{g}-\hat{E}\right) f\left(\hat{P}_{c}\right) \zeta_{s^{\prime}}^{-1}=\mathcal{O}(1)
$$

and

$$
\zeta_{s}^{-1} f(\hat{P})\left[\hat{P}, \hat{\Pi}_{g}\right] \zeta_{s^{\prime}}^{-1}=\mathcal{O}\left(h^{\infty}\right),
$$

uniformly with respect to $h>0$, if $s+s^{\prime}=\delta^{\prime}$ and $1<\delta^{\prime}<\delta$.

Proof: For $g$ as in the theorem, let us choose $\tilde{g} \in C_{0}^{\infty}(\mathbb{R})$ with Supp $\tilde{g} \subset\{g=1\}$ and Supp $f \subset\{\tilde{g}=1\}$ and let us define the selfadjoint operator

$$
\tilde{\Pi}_{g}:=\Pi_{g}-\tilde{g}\left(\hat{P}_{c}\right)\left(E_{g}-\hat{E}\right)-\left(E_{g}-\hat{E}\right) \tilde{g}\left(\hat{P}_{c}\right)+\tilde{g}\left(\hat{P}_{c}\right)\left(E_{g}-\hat{E}\right) \tilde{g}\left(\hat{P}_{c}\right) .
$$

Taking into account Proposition 3.4, we have that $\tilde{\Pi}_{g}=\Pi_{g}+\mathcal{O}\left(h^{\infty}\right)=\Pi_{0}+$ $\mathcal{O}(h)$, and moreover we have

$$
\left(\tilde{\Pi}_{g}-\hat{E}\right) f\left(\hat{P}_{c}\right)=\left(\Pi_{g}-E_{g}\right) f\left(\hat{P}_{c}\right) .
$$

Using Remark 3.3, Proposition 3.4, (3.21) and taking into account that $\left[\hat{P}_{c}, \hat{E}\right]=$ 0 and the fact that $\hat{P}=\hat{P}_{c}+I_{c}(x)$, a straightforward computation gives,

$$
\begin{aligned}
f(\hat{P})\left[\hat{P}, \tilde{\Pi}_{g}\right] & =f(\hat{P})\left[\hat{P}, \Pi_{g}\right]+f(\hat{P})\left[I_{c}(x), \tilde{\Pi}_{g}-\Pi_{g}\right]-f(\hat{P}) \tilde{g}\left(\hat{P}_{c}\right)\left[\hat{P}_{c}, E_{g}\right] \\
& \left.-f(\hat{P})\left[\hat{P}_{c}, E_{g}\right)\right] \tilde{g}\left(\hat{P}_{c}\right)+f(\hat{P}) \tilde{g}\left(\hat{P}_{c}\right)\left[\hat{P}_{c}, E_{g}\right] \tilde{g}\left(\hat{P}_{c}\right)=\mathcal{O}\left(h^{\infty}\right) .(3
\end{aligned}
$$


Moreover, since

$$
\tilde{\Pi}_{g}-\hat{E}-\left(\Pi_{g}-E_{g}\right)=\left(1-\tilde{g}\left(\hat{P}_{c}\right)\right)\left(E_{g}-\hat{E}\right)\left(1-\tilde{g}\left(\hat{P}_{c}\right)\right)
$$

and $f\left(\hat{P}_{c}\right)\left(1-\tilde{g}\left(\hat{P}_{c}\right)\right)=0$, another straightforward computation gives,

$$
\begin{aligned}
f(\hat{P})\left[\hat{P}, \tilde{\Pi}_{g}\right] & =f(\hat{P})\left[\hat{P}, \Pi_{g}\right]-f\left(\hat{P}_{c}\right)\left[\hat{P}_{c}, E_{g}\right] \\
& +\left(f(\hat{P})(\hat{P}+i)-f\left(\hat{P}_{c}\right)\left(\hat{P}_{c}+i\right)\right)(\hat{P}+i)^{-1}\left[\hat{P}, \tilde{\Pi}_{g}-\Pi_{g}\right] \\
& -f\left(\hat{P}_{c}\right) I_{c}(x)(\hat{P}+i)^{-1}\left[\hat{P}, \tilde{\Pi}_{g}-\Pi_{g}\right]+f\left(\hat{P}_{c}\right)\left[I_{c}(x), \tilde{\Pi}_{g}-\Pi_{g}\right] .
\end{aligned}
$$

Let us observe that all the operators involved in the previous formula except $\hat{E}$ are constructed in such a way that, denoting by $A$ one of these operators, we have that, for any $s \in \mathbb{R}$,

$$
\zeta_{s} A \zeta_{s}^{-1}, \quad \zeta_{s}^{-1} A \zeta_{s}
$$

are bounded operators. On the other hand, by taking into account the definition of $\hat{E}$ given in (2.5) and using the exponential decay of the eigenfunctions of $\hat{Q}_{0}(h)$, one can check directly that $\zeta_{s} \hat{E} \zeta_{s}^{-1}$ and $\zeta_{s}^{-1} \hat{E} \zeta_{s}$ are also bounded operators.

Hence, using (3.22) and writing (see: [DiSj, Ma2]),

$f(\hat{P})\left(\hat{P}_{+i}\right)-f\left(\hat{P}_{c}\right)\left(\hat{P}_{c}+i\right)=-\frac{1}{\pi} \int \bar{\partial}(\tilde{f}(z)(z+i))\left(\hat{P}_{-z}\right)^{-1} I_{c}(x)\left(\hat{P}_{c}-z\right)^{-1} d z d \bar{z}$

where $\tilde{f}$ is an almost analytic extension of $f$, we see that

$$
\zeta_{s}^{-1} f(\hat{P})\left[\hat{P}, \tilde{\Pi}_{g}\right] \zeta_{s^{\prime}}^{-1}=\mathcal{O}(1)
$$

if $s+s^{\prime}=\delta^{\prime}<\delta$. Hence by interpolation and commutator estimates (3.28) and (3.30) give

$$
\zeta_{s}^{-1} f(\hat{P})\left[\hat{P}, \tilde{\Pi}_{g}\right] \zeta_{s^{\prime}}^{-1}=\mathcal{O}\left(h^{\infty}\right),
$$

if $s+s^{\prime}=\delta^{\prime}$ and $1<\delta^{\prime}<\delta$. Then, if one set

$$
\hat{\Pi}_{g}:=\frac{1}{2 \pi i} \int_{|z-1|=1 / 2}\left(\tilde{\Pi}_{g}-z\right)^{-1} d z,
$$

we have

$$
\left(\hat{\Pi}_{g}-\hat{E}\right) f\left(\hat{P}_{c}\right)=\frac{i}{2 \pi} \int_{|z-1|=1 / 2}\left(\tilde{\Pi}_{g}-z\right)^{-1}\left(\tilde{\Pi}_{g}-\hat{E}\right) f\left(\hat{P}_{c}\right)(\hat{E}-z)^{-1} d z
$$

and, using (3.27), one can easily obtain (3.24). Moreover, writing

$$
f(\hat{P})\left[\hat{P}, \hat{\Pi}_{g}\right]=\frac{i}{2 \pi} \int_{|z-1|=1 / 2} f(\hat{P})\left(\tilde{\Pi}_{g}-z\right)^{-1}\left[\hat{P}, \tilde{\Pi}_{g}\right]\left(\tilde{\Pi}_{g}-z\right)^{-1} ; d z
$$

and using (3.27), we can conclude that (3.25) holds. 
Corollary 3.7. Under the previous assumptions we also have,

$$
\begin{aligned}
& \left\langle x_{2}-x_{1}\right\rangle^{s}\left(\hat{\Pi}_{g}-\hat{E}\right) f\left(\hat{P}_{c}\right)\left\langle x_{2}-x_{1}\right\rangle^{s^{\prime}}=\mathcal{O}(1), \\
& \left\langle x_{2}-x_{1}\right\rangle^{s} f(\hat{P})\left[\hat{P}, \hat{\Pi}_{g}\right] \hat{\Pi}_{g}\left\langle x_{2}-x_{1}\right\rangle^{s^{\prime}}=\mathcal{O}\left(h^{\infty}\right), \\
& \left\langle x_{2}-x_{1}\right\rangle^{s} \hat{\Pi}_{g} f(\hat{P})\left[\hat{P}, \hat{\Pi}_{g}\right]\left\langle x_{2}-x_{1}\right\rangle^{s^{\prime}}=\mathcal{O}\left(h^{\infty}\right)
\end{aligned}
$$

for any $s+s^{\prime}=\delta^{\prime}, 1<\delta^{\prime}<\delta$, uniformly with respect to $h>0$.

Proof: By commutator estimates and exponential decay of the eigenfunctions of $\tilde{Q}\left(x_{2}-x_{1}\right), \tilde{Q}_{0}$ and $\hat{Q}_{0}(h)$ one has

$$
\zeta_{\delta^{\prime}} \Pi_{0}\left\langle x_{2}-x_{1}\right\rangle^{\delta^{\prime}}, \zeta_{\delta^{\prime}} E_{0}\left\langle x_{2}-x_{1}\right\rangle^{\delta^{\prime}}, \zeta_{\delta^{\prime}} \hat{E}\left\langle x_{2}-x_{1}\right\rangle^{\delta^{\prime}}=\mathcal{O}(1) .
$$

Let us show that we also have

$$
\zeta_{\delta^{\prime}} \hat{\Pi}_{g}\left\langle x_{2}-x_{1}\right\rangle^{\delta^{\prime}}=\mathcal{O}(1) .
$$

Writing

$$
\hat{\Pi}_{g}-\Pi_{0}=\left(\hat{\Pi}_{g}-\Pi_{0}\right)^{2}+\left(\hat{\Pi}_{g}-\Pi_{0}\right) \Pi_{0}+\Pi_{0}\left(\hat{\Pi}_{g}-\Pi_{0}\right),
$$

we obtain that the term

$$
\left\|\zeta_{\delta^{\prime}}\left(\hat{\Pi}_{g}-\Pi_{0}\right)\left\langle x_{2}-x_{1}\right\rangle^{\delta^{\prime}}\right\|
$$

can be estimate by

$$
\begin{aligned}
& \left\|\zeta_{\delta^{\prime}}\left(\hat{\Pi}_{g}-\Pi_{0}\right)\left\langle x_{2}-x_{1}\right\rangle^{\delta^{\prime}}\right\|\left\|\left\langle x_{2}-x_{1}\right\rangle^{-\delta^{\prime}}\left(\hat{\Pi}_{g}-\Pi_{0}\right)\left\langle x_{2}-x_{1}\right\rangle^{\delta^{\prime}}\right\| \\
& +\left\|\zeta_{\delta^{\prime}}\left(\hat{\Pi}_{g}-\Pi_{0}\right) \zeta_{\delta^{\prime}}^{-1}\right\|\left\|\zeta_{\delta^{\prime}} \Pi_{0}\left\langle x_{2}-x_{1}\right\rangle^{\delta^{\prime}}\right\| \\
& +\left\|\zeta_{\delta^{\prime}} \Pi_{0}\left\langle x_{2}-x_{1}\right\rangle^{\delta^{\prime}}\right\|\left\|\left\langle x_{2}-x_{1}\right\rangle^{-\delta^{\prime}}\left(\hat{\Pi}_{g}-\Pi_{0}\right)\left\langle x_{2}-x_{1}\right\rangle^{\delta^{\prime}}\right\| .
\end{aligned}
$$

Since $\left\|\left\langle x_{2}-x_{1}\right\rangle^{-\delta^{\prime}}\left(\hat{\Pi}_{g}-\Pi_{0}\right)\left\langle x_{2}-x_{1}\right\rangle^{\delta^{\prime}}\right\|=\mathcal{O}(h)$, if $h$ is sufficiently small, then this term can be taken less than $1 / 2$. Hence, one obtains that (3.37) can be estimate by

$$
\begin{aligned}
& 2\left\|\zeta_{\delta^{\prime}}\left(\hat{\Pi}_{g}-\Pi_{0}\right) \zeta_{\delta^{\prime}}^{-1}\right\|\left\|\zeta_{\delta^{\prime}} \Pi_{0}\left\langle x_{2}-x_{1}\right\rangle^{\delta^{\prime}}\right\| \\
& +\left\|\zeta_{\delta^{\prime}} \Pi_{0}\left\langle x_{2}-x_{1}\right\rangle^{\delta^{\prime}}\right\|\left\|\left\langle x_{2}-x_{1}\right\rangle^{-\delta^{\prime}}\left(\hat{\Pi}_{g}-\Pi_{0}\right)\left\langle x_{2}-x_{1}\right\rangle^{\delta^{\prime}}\right\|
\end{aligned}
$$

and it is easy to check that (3.38) is uniformly bounded. Hence, writing

$$
\zeta_{\delta^{\prime}} \hat{\Pi}_{g}\left\langle x_{2}-x_{1}\right\rangle^{\delta^{\prime}}=\zeta_{\delta^{\prime}} \Pi_{0}\left\langle x_{2}-x_{1}\right\rangle^{\delta^{\prime}}+\zeta_{\delta^{\prime}}\left(\hat{\Pi}_{g}-\Pi_{0}\right)\left\langle x_{2}-x_{1}\right\rangle^{\delta^{\prime}}
$$

we obtain (3.36). Since

$$
\hat{\Pi}_{g}-\hat{E}=\left(\hat{\Pi}_{g}-\hat{E}\right) \hat{\Pi}_{g}+\hat{E}\left(\hat{\Pi}_{g}-\Pi_{0}\right),
$$

using (3.24), (3.36) and commutator estimates, one also obtains (3.33). Moreover (3.25) (3.36) and commutator estimates, give (3.34) and (3.35).

Let us denote by $\hat{\Pi}_{g}^{\perp}=1-\hat{\Pi}_{g}$ and let us define $\hat{P}^{A D}=\hat{\Pi}_{g} \hat{P} \hat{\Pi}_{g}$. We have the following result: 
Theorem 3.8. Under the same assumptions of Theorem 3.6, there exist two bounded operators $\mathcal{W}, \mathcal{W}_{0}: L^{2}\left(\mathbb{R}^{(2+N) n}\right) \rightarrow L^{2}\left(\mathbb{R}^{2 n}\right)^{\oplus m}$ such that

$$
\begin{array}{ll}
\mathcal{W W}^{*}=1, & \mathcal{W}^{*} \mathcal{W}=\hat{\Pi}_{g} \\
\mathcal{W}_{0} \mathcal{W}_{0}^{*}=1, & \mathcal{W}_{0}^{*} \mathcal{W}_{0}=\hat{E}
\end{array}
$$

and

$$
\left\|\left\langle x_{2}-x_{1}\right\rangle^{s}\left(\mathcal{W}-\mathcal{W}_{0}\right) f\left(\hat{P}_{c}\right)\left\langle x_{2}-x_{1}\right\rangle^{s^{\prime}}\right\|=\mathcal{O}(1)
$$

if $s+s^{\prime}=\delta^{\prime}$, for $1<\delta^{\prime}<\delta$.

The operators $\hat{A}:=\mathcal{W} \hat{P} \mathcal{W}^{*}=\mathcal{W} \hat{P}^{A D} \mathcal{W}^{*}$ and $\hat{A}_{0}:=\mathcal{W}_{0} \hat{P}_{c} \mathcal{W}_{0}^{*}$ are $h$-admissible operator on $L^{2}\left(\mathbb{R}^{2 n}\right)^{\oplus m}$ with domain $H^{2}\left(\mathbb{R}^{2 n}\right)^{\oplus m}$, and their symbols $\hat{a}:=$ $\hat{a}\left(x_{2}-x_{1}, \xi ; h\right)$ and $\hat{a}_{0}:=\hat{a}_{0}(\xi ; h)$, verify:

$$
\begin{aligned}
\hat{a}\left(x_{2}-x_{1}, \xi ; h\right) & =\frac{1}{2}\left(\xi_{1}-\xi_{2}\right)^{2} \mathbf{I}_{m}+\Lambda\left(x_{2}-x_{1}\right)+h r\left(x_{2}-x_{1}, \xi ; h\right)(3.4 \\
\hat{a}_{0}(\xi ; h) & =\frac{1}{2}\left(\xi_{1}-\xi_{2}\right)^{2} \mathbf{I}_{m}+\mathcal{E}_{0}+h r_{c}(\xi ; h) .
\end{aligned}
$$

where

- $\Lambda\left(x_{2}-x_{1}\right)$ is a $m \times m$ matrix depending smoothly on $x_{2}-x_{1}$, with spectrum $\sigma\left(x_{2}-x_{1}\right)$,

- $\mathcal{E}_{0}$ is a $m \times m$ matrix with spectrum $\sigma_{0}$

- $\left\langle x_{2}-x_{1}\right\rangle^{s}\left(\hat{A}-\hat{A}_{0}\right) f\left(\hat{A}_{0}\right)\left\langle x_{2}-x_{1}\right\rangle^{s^{\prime}}=\mathcal{O}(1)$, if $s+s^{\prime}=\delta^{\prime}$ and $1<\delta^{\prime}<\delta$;

- $r\left(x_{2}-x_{1}, \xi ; h\right)$ and $r_{c}(\xi ; h)$ verify:

$$
\partial^{\alpha} r\left(x_{2}-x_{1}, \xi ; h\right), \partial^{\alpha} r_{c}(\xi ; h)=\mathcal{O}(\langle\xi\rangle)
$$

for any multi-index $\alpha$ and uniformly with respect to $(x, \xi) \in T^{*} \mathbb{R}^{2 n}$ and $h>0$ small enough.

Proof: Since $\hat{\Pi}_{g}-\Pi_{0}=\mathcal{O}(h)$ and $\hat{E}-E_{0}=\mathcal{O}(h)$, for $h$ small enough we can consider the unitary operators $\mathcal{V}$ and $\mathcal{V}_{0}$ on $L^{2}\left(\mathbb{R}^{(2+N) n}\right)$ defined by the Nagy formula,

$$
\begin{aligned}
\mathcal{V} & =\left(\Pi_{0} \hat{\Pi}_{g}+\left(1-\Pi_{0}\right)\left(1-\hat{\Pi}_{g}\right)\right)\left(1-\left(\hat{\Pi}_{g}-\Pi_{0}\right)^{2}\right)^{-1 / 2}, \\
\mathcal{V}_{0} & =\left(E_{0} \hat{E}+\left(1-E_{0}\right)(1-\hat{E})\right)\left(1-\left(\hat{E}-E_{0}\right)^{2}\right)^{-1 / 2} .
\end{aligned}
$$

We have (see: [Ka] Chap.I.4 and [MaSo2]),

$$
\mathcal{V}^{*} \mathcal{V}=\mathcal{V} \mathcal{V}^{*}=1, \quad \mathcal{V}_{0}^{*} \mathcal{V}_{0}=\mathcal{V}_{0} \mathcal{V}_{0}^{*}=1 \quad \text { and } \quad \Pi_{0} \mathcal{V}=\mathcal{V} \hat{\Pi}_{g}, \quad E_{0} \mathcal{V}_{0}=\mathcal{V}_{0} \hat{E}
$$

Observe that, by (2.9) and (3.33)

$$
\left\|\left\langle x_{2}-x_{1}\right\rangle^{s}\left(\mathcal{V}-\mathcal{V}_{0}\right) f\left(\hat{P}_{c}\right)\left\langle x_{2}-x_{1}\right\rangle^{s^{\prime}}\right\|=\mathcal{O}(1)
$$


if $s+s^{\prime}=\delta^{\prime}$, for $1<\delta^{\prime}<\delta$.

Let us define $Z: L^{2}\left(\mathbb{R}^{2 n} ; L^{2}\left(\mathbb{R}^{n N}\right)\right) \rightarrow L^{2}\left(\mathbb{R}^{2 n}\right)^{\oplus m}$ by,

$$
Z \psi(x)=\left(\left\langle\psi(x), u_{1}(x)\right\rangle_{L^{2}\left(\mathbb{R}^{n N}\right)}, \ldots,\left\langle\psi(x), u_{m}(x)\right\rangle_{L^{2}\left(\mathbb{R}^{n N}\right)}\right.
$$

where $u_{j}(x)$, are the eigenvalue of $Q(x)$ associated to $\lambda_{j}\left(x_{2}-x_{1}\right), j=1, \ldots, m$, and $Z_{0}: L^{2}\left(\mathbb{R}^{2 n} ; L^{2}\left(\mathbb{R}^{n N}\right)\right) \rightarrow L^{2}\left(\mathbb{R}^{2}\right)^{\oplus m}$ by,

$$
Z_{0} \psi(x)=\left(\left\langle\psi(x), u_{1}^{0}(x)\right\rangle_{L^{2}\left(\mathbb{R}^{n N}\right)}, \ldots,\left\langle\psi(x), u_{m}^{0}(x)\right\rangle_{L^{2}\left(\mathbb{R}^{n N}\right)}\right.
$$

where $u_{j}^{0}(x)$, are the eigenvalue $Q_{0}(x)$ associated to $e_{j}, j=1, \ldots, m$, i.e. $u_{j}^{0}(x)=$ $S_{x} \tilde{u}_{j}^{0}$ where $\tilde{u}_{j}^{0}$ are the eigenvalue of $\tilde{Q}_{0}$. Moreover, let us set

$$
\mathcal{W}:=Z \circ \mathcal{V}=Z_{L}+\mathcal{O}(h), \quad \mathcal{W}_{0}:=Z_{0} \circ \mathcal{V}=Z_{0}+\mathcal{O}(h) .
$$

Proposition 2.1 and (3.46) give (3.41).

Denoting by

$$
\begin{aligned}
& S_{k}: L^{2}\left(\mathbb{R}^{2 n}\right) \rightarrow L^{2}\left(\mathbb{R}^{2 n}\right), \\
& S_{k} \phi\left(x_{1}, x_{2}\right)=\phi\left(x_{1}+k, x_{2}+k\right), \quad k \in \mathbb{R}^{3}
\end{aligned}
$$

and

$$
\begin{aligned}
& S_{k_{1}, k_{2}}^{c}: L^{2}\left(\mathbb{R}^{2 n}\right) \rightarrow L^{2}\left(\mathbb{R}^{2 n}\right), \\
& S_{k_{1}, k_{2}}^{c} \phi\left(x_{1}, x_{2}\right)=\phi\left(x_{1}+k_{1}, x_{2}+k_{2}\right), \quad k_{1}, k_{2} \in \mathbb{R}^{3}
\end{aligned}
$$

then, since $T_{k} u_{j}=u_{j}$ and $T_{k_{1}, k_{2}}^{c} u_{j}^{0}=u_{j}^{0}$ for any $j=1, \ldots, m$,

$$
S_{k} \mathcal{W}=\mathcal{W}\left(T_{k} \oplus . . \oplus S_{k}\right), \quad S_{k_{1}, k_{2}}^{c} \mathcal{W}_{0}=\mathcal{W}_{0}\left(T_{k_{1}, k_{2}}^{c} \oplus . . \oplus S_{k_{1}, k_{2}}^{c}\right) .
$$

Thanks to the properties of $\mathcal{V}$, we see that $\mathcal{W} \hat{\Pi}_{g}=\mathcal{W}, \mathcal{W}_{0} \hat{E}=\mathcal{W}_{0}$ and, since $Z^{*} Z=\Pi_{0}, Z_{0}^{*} Z_{0}=E_{0}$ and $Z Z^{*}=Z_{0} Z_{0}^{*}=1$, we also obtain:

$$
\mathcal{W}^{*} \mathcal{W}=\hat{\Pi}_{g} ; \mathcal{W W}^{*}=1, \quad \mathcal{W}_{0}^{*} \mathcal{W}_{0}=\hat{E} ; \mathcal{W}_{0} \mathcal{W}_{0}^{*}=1
$$

Then, defining

$$
\hat{A}:=\mathcal{W} \hat{P} \mathcal{W}^{*}=\mathcal{W} \hat{P}_{g}^{A D} \mathcal{W}^{*}, \quad \hat{A}_{0}:=\mathcal{W}_{0} \hat{P}_{c} \mathcal{W}_{0}^{*}
$$

one easily obtains that $\hat{A}$ and $\hat{A}_{0}$ are operators on $L^{2}\left(\mathbb{R}^{2 n}\right)^{\oplus m}$ that commute with $S_{k}^{\oplus m}$. This proves that $a$ depends only on $x_{2}-x_{1}$ and that $A_{0}$ is independent of $x$. An easy calculation (see also Chapter 10 in[MaSo2]) gives the principal term in the asymptotic expansion of the symbols $\hat{a}$ and $\hat{a}_{0}$ and estimates on all the derivatives of the remainder term $r$ and $r_{c}$. More precisely, by using (3.41) one can also prove that

$$
\hat{A}-\hat{A}_{0}=\Lambda\left(x_{2}-x_{1}\right)-\varepsilon_{0}+\sum_{|\alpha|=1} b_{\alpha}\left(x_{2}-x_{1}\right)\left(h D_{x}\right)^{\alpha}+Q\left(x_{2}-x_{1}, h D_{x}\right)
$$

with

$\left\langle x_{2}-x_{1}\right\rangle^{s} b_{\alpha}\left(x_{2}-x_{1}\right)\left\langle x_{2}-x_{1}\right\rangle^{s^{\prime}},\left\langle x_{2}-x_{1}\right\rangle^{s} Q\left(x_{2}-x_{1}, h D_{x}\right)\left\langle x_{2}-x_{1}\right\rangle^{s^{\prime}}=\mathcal{O}(1)$ for any if $s+s^{\prime}=\delta^{\prime}$, for $1<\delta^{\prime}<\delta$. 


\section{Born-Oppenheimer approximation of wave operators}

Let $f \in C_{0}^{\infty}(\mathbb{R})$ and consider the wave operators

$$
\begin{aligned}
& \Omega_{ \pm}^{A D} f\left(\hat{P}_{c}\right):=s-\lim _{t \rightarrow \pm \infty} e^{i t \hat{P}^{A D}} e^{-i t \hat{P}_{c}} \hat{E} f\left(\hat{P}_{c}\right) \\
& \Omega_{ \pm}^{N A D}:=s-\lim _{t \rightarrow \pm \infty} e^{i t \hat{P}} e^{-i t \hat{P}^{A D}} \hat{\Pi}_{g} f\left(\hat{P}^{A D}\right) .
\end{aligned}
$$

We have the following:

Theorem 4.1. Let $f \in C_{0}^{\infty}(\mathbb{R})$ such that Supp $f \subset\{g=1\}$. Under the previous assumptions, the wave operators $\Omega_{ \pm}^{A D}, \Omega_{ \pm}^{N A D}$ exist, are complete and,

$$
\Omega_{ \pm}^{c} \hat{E} f\left(\hat{P}_{c}\right)=\Omega_{ \pm}^{N A D} \Omega_{ \pm}^{A D} \hat{E} f\left(\hat{P}_{c}\right)
$$

Proof: Let us start by proving the existence of $\Omega_{ \pm}^{A D}$. Since, by (3.33),

$$
\begin{aligned}
\hat{\Pi}_{g}^{\perp} e^{i t \hat{P}^{A D}} e^{-i t \hat{P}_{c}} \hat{E} f\left(\hat{P}_{c}\right) & =\hat{\Pi}_{g}^{\perp} e^{-i t \hat{P}_{c}} \hat{E} f\left(\hat{P}_{c}\right)=-\left(\hat{\Pi}_{g}-\hat{E}\right) f\left(\hat{P}_{c}\right) e^{-i t \hat{P}_{c}} \hat{E} \\
& =\mathcal{O}\left(\left\langle x_{2}-x_{1}\right\rangle^{-\delta^{\prime}}\right) e^{-i t \hat{P}_{c}} \hat{E}
\end{aligned}
$$

for any $\delta^{\prime}, 1<\delta^{\prime}<\delta$, and $\left\langle x_{2}-x_{1}\right\rangle^{-\delta^{\prime}} \mathcal{W}_{0}^{*}\left\langle x_{2}-x_{1}\right\rangle^{\delta^{\prime}}=\mathcal{O}(1)$, then

$$
\begin{aligned}
\left\|\hat{\Pi}_{g}^{\perp} e^{i t \hat{P}^{A D}} e^{-i t \hat{P}_{c}} \hat{E} f\left(\hat{P}_{c}\right)\right\| & \leq C_{1}\left\|\left\langle x_{2}-x_{1}\right\rangle^{-\delta^{\prime}} e^{-i t \hat{P}_{c}} f\left(\hat{P}_{c}\right) \phi\right\| \\
& \leq C_{2}\left\|\left\langle x_{2}-x_{1}\right\rangle^{-\delta^{\prime}} e^{-i t \hat{A}_{0}} f\left(\hat{A}_{0}\right) \mathcal{W}_{0} \phi\right\|
\end{aligned}
$$

Denoting by $\tilde{U}$ the change of variable

$$
X=\frac{1}{2}\left(x_{1}-x_{2}\right), \quad R=\frac{1}{2}\left(x_{1}+x_{2}\right),
$$

by $\mathcal{F}$ the Fourier transform with respect the $R$ variable

$$
(\mathcal{F} f)(X, \rho)=(2 \pi)^{-n / 2} \int e^{-i R \rho} f(X, R) d R
$$

and setting

$$
\hat{B}_{0}:=\tilde{U} \mathcal{F}^{-1} \hat{A}_{0} \mathcal{F} \tilde{U}^{-1}=-\frac{h^{2}}{2} \Delta_{X}+\mathcal{E}_{0}+h r_{c}\left(h D_{X}, \rho ; h\right)
$$

then we have

$$
\left\langle x_{2}-x_{1}\right\rangle^{-\delta} e^{-i t \hat{A}_{0}} f\left(\hat{A}_{0}\right) \mathcal{W}_{0}=\tilde{U} \mathcal{F}^{-1}\langle X\rangle^{-\delta} e^{-i t \hat{B}_{0}} f\left(\hat{B}_{0}\right) \mathcal{F} \tilde{U}^{-1} \mathcal{W}_{0} .
$$

Hence, for $\phi$ in a dense subset of $L^{2}\left(\mathbb{R}^{n(2+N)}\right)$,

$$
\left\|\langle X\rangle^{-\delta^{\prime}} e^{-i t \hat{B}_{0}} f\left(\hat{B}_{0}\right) \tilde{U} \mathcal{W}_{0} \phi\right\| \leq C(\phi)\langle t\rangle^{-\delta^{\prime}} .
$$


and then from (4.1), (4.4) and (4.5), we obtain

$$
s-\lim _{t \rightarrow \pm \infty} \hat{\Pi}_{g}^{\perp} e^{i t \hat{P}^{A D}} e^{-i t \hat{P}_{c}} \hat{E} f\left(\hat{P}_{c}\right)=0 .
$$

Therefore, we can reduced to prove the existence of

$$
\Omega_{ \pm}^{A D} f\left(\hat{P}_{c}\right)=s-\lim _{t \rightarrow \pm \infty} \hat{\Pi}_{g} e^{i t \hat{P}^{A D}} e^{-i t \hat{P}_{c}} \hat{E} f\left(\hat{P}_{c}\right) .
$$

We have,

$$
\begin{aligned}
\hat{\Pi}_{g} e^{i t \hat{P}^{A D}} e^{-i t \hat{P}_{c}} \hat{E} f\left(\hat{P}_{c}\right) & =\mathcal{W}^{*} \mathcal{W} e^{i t \hat{P}^{A D}} \mathcal{W W}_{0}^{*} e^{-i t \hat{P}_{c}} \hat{E} f\left(\hat{P}_{c}\right) \\
& +\hat{\Pi}_{g} e^{i t \hat{P}^{A D}}\left(1-\mathcal{W} \mathcal{W}_{0}^{*}\right) \hat{E} e^{-i t \hat{P}_{c}} f\left(\hat{P}_{c}\right) .
\end{aligned}
$$

As before, since (see (3.41)) $\left\langle x_{2}-x_{1}\right\rangle^{\delta^{\prime}}\left(1-\mathcal{W} \mathcal{W}_{0}^{*}\right) \hat{E}=\mathcal{O}(1)$, we get, for $\phi$ in a dense subset of $L^{2}\left(\mathbb{R}^{n(N+2)}\right)$, that

$$
\left\|\hat{\Pi}_{g} e^{i t \hat{P}^{A D}}\left(1-\mathcal{W} \mathcal{W}_{0}^{*}\right) \hat{E} e^{-i t \hat{P}_{c}} f\left(\hat{P}_{c}\right) \phi\right\| \leq C(\phi)\langle t\rangle^{-\delta^{\prime}}
$$

and then

$$
s-\lim _{t \rightarrow \pm \infty} \hat{\Pi}_{g} e^{i t \hat{P}^{A D}}\left(1-\mathcal{W} \mathcal{W}_{0}^{*}\right) \hat{E} e^{-i t \hat{P}_{c}} f\left(\hat{P}_{c}\right)=0 .
$$

In conclusion, from (4.6) and (4.7) we obtain

$$
\Omega_{ \pm}^{A D} f\left(\hat{P}_{c}\right)=\mathcal{W}^{*} \tilde{\Omega}_{ \pm} f\left(\hat{A}_{0}\right) \mathcal{W}_{0}
$$

where

$$
\tilde{\Omega}_{ \pm} f\left(\hat{A}_{0}\right)=s-\lim _{t \rightarrow \pm \infty} e^{i t \hat{A}} e^{-i t \hat{A}_{0}} f\left(\hat{A}_{0}\right) .
$$

Taking into account that $\hat{A}$ is unitary equivalent (cfr.: (4.2) and (4.3)) to the operator

$$
\hat{B}=\tilde{U} \mathcal{F}^{-1} \hat{A} \mathcal{F} \tilde{U}^{-1}=-h^{2} \Delta_{X}+\Lambda(X)+h r\left(h D_{X}, \rho ; h\right),
$$

acting on $L^{2}(\mathbb{R})$ and depending on $\rho \in \mathbb{R}$ as a parameter, and applying classical Mourre estimates $([\mathrm{Mo}])$, one can obtain the existence and completeness of $\tilde{\Omega}_{ \pm}$ and then the one of $\Omega_{ \pm}^{A D}$.

To study $\Omega_{ \pm}^{N A D}$ let us observe that one can write

$$
\Omega_{ \pm}^{N A D} f\left(\hat{P}^{A D}\right)=s-\lim _{t \rightarrow \pm \infty} f(\hat{P}) e^{i t \hat{P}} e^{-i t \hat{P}^{A D}} .
$$

Then

$$
\frac{d}{d t}\left(f(\hat{P}) e^{i t \hat{P}} e^{-i t \hat{P}^{A D}} \hat{\Pi}_{g}\right)=e^{i t \hat{P}} f(\hat{P})\left(\hat{P}-\hat{\Pi}_{g} \hat{P} \hat{\Pi}_{g}\right) \hat{\Pi}_{g} e^{-i t \hat{P}^{A D}}
$$

By using (3.34) we obtain

$$
f(\hat{P})\left(\hat{P}-\hat{\Pi}_{g} \hat{P} \hat{\Pi}_{g}\right) \hat{\Pi}_{g}=f(\hat{P})\left[\hat{P}, \hat{\Pi}_{g}\right] \hat{\Pi}_{g}=\mathcal{O}\left(h^{\infty}\left\langle x_{2}-x_{1}\right\rangle^{-\delta^{\prime}}\right) \hat{\Pi}_{g}
$$


and thenwe get the existence of the wave operators $\Omega_{ \pm}^{N A D}$. Since

$$
\Omega_{ \pm}^{c} \hat{E} f\left(\hat{P}_{c}\right)=\Omega_{ \pm}^{N A D} \Omega_{ \pm}^{A D} f\left(\hat{P}_{c}\right)
$$

then $R\left(\Omega_{ \pm}^{c}\right) \subset R\left(\Omega_{ \pm}^{N A D}\right)$. Moreover, $\phi=\Omega_{ \pm}^{N A D} \psi$ with $\psi \in R\left(\hat{\Pi}_{g}\right)$ then there exists $\theta \in R(\hat{E})$ such that $\psi=\Omega_{ \pm}^{A D} \theta$ and then $\phi=\Omega_{ \pm}^{c} \theta$.

\section{The adiabatic wave operators}

In the following we assume that $m=1$. In such a case, the computation given in Chapter 10 of [MaSo2] shows that in the operators $\hat{A}$ and $\hat{A}_{0}$ the terms of order $h$ are absent, and then, they can be rewritten as

$$
\begin{aligned}
\hat{A} & =\frac{h^{2}}{2}\left(D_{x_{1}}-D_{x_{2}}\right)^{2}+\lambda_{1}\left(x_{2}-x_{1}\right)+h^{2} L\left(x_{2}-x_{1}, h D_{x_{1}}, h D_{x_{2}} ; h\right) \\
\hat{A}_{0} & =\frac{h^{2}}{2}\left(D_{x_{1}}-D_{x_{2}}\right)^{2}+e_{1}+h^{2} L_{0}\left(h D_{x_{1}}, h D_{x_{2}} ; h\right)
\end{aligned}
$$

for some bounded operators $L$ and $L_{0}$. Let us assume us assume also that a non trapping condition holds i.e. :

(H3) The classical Hamiltonian $\frac{1}{2} \Xi^{2}+\lambda_{1}(X), X \in \mathbb{R}^{n}$, is non trapping at the energy $\mu_{0}$.

Next, let us denote by $R(z ; h)=(\hat{P}-z)^{-1}$, by $R^{A D}(z ; h)=\left(\hat{P}^{A D}-z\right)^{-1} \hat{\Pi}_{g}$ and by $R^{\perp}(z ; h)=\left(\hat{\Pi}_{g}^{\perp} \hat{P} \hat{\Pi}_{g}^{\perp}-z\right)^{-1} \hat{\Pi}_{g}^{\perp}$ the resolvents of $\hat{P}, \hat{P}^{A D}$ and $\hat{\Pi}_{g}^{\perp} P \hat{\Pi}_{g}^{\perp}$ respectively. Let us estimate the boundary value of the resolvents, by Mourre's commutator method.

Proposition 5.1. Under assumptions $(H 1),(H 2)$ and (H3), if $\mu_{0} \in \mathbb{R}$ satisfy

$$
\mu_{0}<\inf _{x} \inf \left(\operatorname{Sp}\left(\tilde{Q}\left(x_{2}-x_{1}\right) \backslash \lambda_{1}\left(x_{2}-x_{1}\right)\right)\right.
$$

then there exists $\varepsilon>0$ such that, if $\phi \in C_{0}^{\infty}\left(\mu_{0}-\varepsilon, \mu_{0}+\varepsilon\right)$, Supp $\phi \subset\{g=1\}$, and, if $\frac{1}{2}<s<\frac{\delta^{\prime}}{2}, \delta^{\prime}<\delta$, and $\lambda \in\left(\mu_{0}-\varepsilon, \mu_{0}+\varepsilon\right)$ we have that

$$
\left\|\left\langle x_{2}-x_{1}\right\rangle^{-s} R^{A D}(\lambda \pm i 0)\left\langle x_{2}-x_{1}\right\rangle^{-s}\right\| \leq C h^{-1}
$$

and

$$
\left\|\left\langle x_{2}-x_{1}\right\rangle^{-s} R(\lambda \pm i 0) \phi(\hat{P})\left\langle x_{2}-x_{1}\right\rangle^{-s}\right\| \leq C h^{-1}
$$

for some constant $C>0$ independent of $h$.

Proof: Assumption (H3) guarantees that there exists a conjugate operator of $\hat{B}$ defined in (4.9)and then, for $s \in \mathbb{R}, s>\frac{1}{2}$, one has

$$
\left\|\left\langle x_{2}-x_{1}\right\rangle^{-s}(\hat{B}-\lambda \pm i 0)^{-1}\left\langle x_{2}-x_{1}\right\rangle^{-s}\right\| \leq C h^{-1}
$$


for $\lambda \in\left(\mu_{0}-\varepsilon, \mu_{0}+\varepsilon\right)$, for some $\varepsilon>0$ and then, since $\hat{B}$ is unitary equivalent to $\hat{P}^{A D}$, we obtain (5.1). Now, as in Theorem 3.4 of [KMW1] one can write

$$
R(z ; h)=R^{A D}(z ; h)+R^{\perp}(z ; h)-\left(M(z, h)+M(\bar{z} ; h)^{*}\right)+R(z ; h) T(z, h)
$$

where

$$
\begin{aligned}
M(z, h) & =R^{\perp}(z ; h) \hat{P} R^{A D}(z ; h) \\
T(z ; h) & =\hat{\Pi}_{g} \hat{P} \hat{\Pi}_{g}^{\perp} M(z, h)+\hat{\Pi}_{g}^{\perp} \hat{P} \hat{\Pi}_{g} M(\bar{z} ; h)^{*}
\end{aligned}
$$

Let us choose $\phi_{1}, \psi_{1}, \phi_{2}, \in C_{0}^{\infty}\left(\mu_{0}-\varepsilon, \mu_{0}+\varepsilon\right)$, such that $\phi_{1} \phi=\phi, \psi_{1} \phi=\phi$, $\phi_{2} \phi_{1}=\phi_{1}$, Supp $\phi_{2} \subset\{g=1\}$ and let us write $\phi_{1}(\hat{P}) T(z ; h)$, as,

$$
\begin{array}{r}
\phi_{1}(\hat{P}) T(z ; h)=-\left(\phi_{2}(\hat{P}) \hat{\Pi}_{g} \phi_{1}(\hat{P})\left[\hat{P}, \hat{\Pi}_{g}\right]+\phi_{2}(\hat{P})\left[\phi_{1}(\hat{P}), \hat{\Pi}_{g}\right]\left[\hat{P}, \hat{\Pi}_{g}\right]\right) M(z, h) \\
+\phi_{1}(\hat{P})\left[\hat{P}, \hat{\Pi}_{g}\right] M(\bar{z}, h)^{*}
\end{array}
$$

Then, by (3.34) and (3.35), one obtain

$$
\left\langle x_{2}-x_{1}\right\rangle^{s} \phi_{1}(P) T(z ; h) \psi_{1}(\hat{P})\left\langle x_{2}-x_{1}\right\rangle^{-s}=\mathcal{O}\left(h^{\infty}\right)
$$

uniformly in $z=\lambda+i \theta, \theta \neq 0$. Hence

$$
\begin{aligned}
\phi(\hat{P}) R(z ; h) & =\phi(\hat{P})\left(R^{A D}(z ; h)+R^{\perp}(z ; h)-\left(M(z, h)+M(z ; h)^{*}\right)\right) \\
& \times \psi_{1}(\hat{P})\left(1-\phi_{1}(\hat{P}) T(z, h) \psi_{1}(\hat{P})\right)^{-1}
\end{aligned}
$$

Moreover, we have

$$
R^{\perp}(z ; h)=\mathcal{O}(1)
$$

uniformly in $h>0$, for $z$ in a small complex neighborhood of $\mu_{0}$ and then from (5.4) and (5.1) we get (5.2)

Proposition 5.2. Under assumptions (H1), (H2) and (H3), if $\mu_{0} \in \mathbb{R}$ satisfy

$$
\mu_{0}<\inf _{x} \inf \left(\operatorname{Sp}\left(\tilde{Q}\left(x_{2}-x_{1}\right) \backslash \lambda_{1}\left(x_{2}-x_{1}\right)\right)\right.
$$

then there exists $\varepsilon>0$ such that, if $\phi \in C_{0}^{\infty}\left(\mu_{0}-\varepsilon, \mu_{0}+\varepsilon\right)$, Supp $\phi \subset\{g=1\}$, and $\frac{1}{2}<s<\frac{\delta^{\prime}}{2}, \delta^{\prime}<\delta$,

$$
\begin{aligned}
& \int_{-\infty}^{+\infty}\left\|\left\langle x_{2}-x_{1}\right\rangle^{-s} e^{-i t \hat{P}^{A D} / h} \phi\left(\hat{P}^{A D}\right) f\right\|^{2} \leq C\|f\|^{2} \\
& \int_{-\infty}^{+\infty}\left\|\left\langle x_{2}-x_{1}\right\rangle^{-s} e^{-i t \hat{P} / h} \phi(\hat{P}) f\right\|^{2} \leq C\|f\|^{2}
\end{aligned}
$$

for all $f \in L^{2}\left(\mathbb{R}^{n(N+2)}\right)$, uniformly in $h$.

Proof: The Proposition is a consequence of the resolvent estimates (5.1) and (5.2), and the arguments of [Wa], Lemma 3.3.

We have the following: 
Lemma 5.3. Let $\psi, \psi_{1} \in C_{0}^{\infty}(\mathbb{R})$, Supp $\psi$, Supp $\psi_{1} \subset\{g=1\}$. Then we have

$$
\left\|\left\langle x_{2}-x_{1}\right\rangle^{s}\left(\psi\left(\hat{P}^{A D}\right)-\psi(\hat{P})\right) \psi_{1}\left(\hat{P}^{A D}\right) \hat{\Pi}_{g}\left\langle x_{2}-x_{1}\right\rangle^{s^{\prime}}\right\|=\mathcal{O}\left(h^{\infty}\right)
$$

if $s+s^{\prime}=\delta^{\prime}$ and $1<\delta^{\prime}<\delta$.

Proof: At first, let us observe that, for any $\phi, \phi_{1} \in C_{0}^{\infty}(\mathbb{R})$, Supp $\phi, \phi_{1} \subset\{g=$ $1\}$, one has

$$
\left\langle x_{2}-x_{1}\right\rangle^{s}\left(\phi\left(\hat{P}^{A D}\right)-\phi(\hat{P})\right) \hat{\Pi}_{g} \phi_{1}(\hat{P}) \hat{\Pi}_{g}\left\langle x_{2}-x_{1}\right\rangle^{s^{\prime}}=\mathcal{O}\left(h^{\infty}\right)
$$

for $s+s^{\prime}=\delta^{\prime}<\delta$. Actually, one can write

$$
\left(\phi\left(\hat{P}^{A D}\right)-\phi(\hat{P})\right) \hat{\Pi}_{g}=-\frac{1}{\pi} \int \bar{\partial} \tilde{\phi}(z)\left(R(z)-R^{A D}(z)\right) \hat{\Pi}_{g} d z d \bar{z}
$$

where $\tilde{\phi}$ is an almost analytic extension of $\phi$, and then, one has

$$
\begin{aligned}
& \left\|\left\langle x_{2}-x_{1}\right\rangle^{s}\left(\phi\left(\hat{P}^{A D}\right)-\phi(\hat{P})\right) \hat{\Pi}_{g} \phi_{1}(\hat{P}) \hat{\Pi}_{g}\left\langle x_{2}-x_{1}\right\rangle^{s^{\prime}}\right\| \\
& \leq C\left\|\left\langle x_{2}-x_{1}\right\rangle^{s}\left[P, \hat{\Pi}_{g}\right] \hat{\Pi}_{g} \phi_{1}(\hat{P}) \hat{\Pi}_{g}\left\langle x_{2}-x_{1}\right\rangle^{s^{\prime}}\right\| .
\end{aligned}
$$

Hence, writing

$$
\left[P, \hat{\Pi}_{g}\right] \hat{\Pi}_{g} \phi_{1}(\hat{P}) \hat{\Pi}_{g}=\left[P, \hat{\Pi}_{g}\right] \phi_{1}(\hat{P}) \hat{\Pi}_{g}-\hat{\Pi}_{g}\left[P, \hat{\Pi}_{g}\right] \phi_{1}(\hat{P}) \hat{\Pi}_{g}
$$

and applying (3.34) and (3.35), one obtains

$$
\left\langle x_{2}-x_{1}\right\rangle^{s}\left[P, \hat{\Pi}_{g}\right] \hat{\Pi}_{g} \phi_{1}(\hat{P})\left\langle x_{2}-x_{1}\right\rangle^{s^{\prime}}=\mathcal{O}\left(h^{\infty}\right)
$$

and then, by (5.8), one obtains (5.7).

Let us now choose $\psi_{2}, . ., \psi_{N} \in C_{0}^{\infty}(\mathbb{R})$ such that $\psi_{j} \psi_{j+1}=\psi_{j}$ for any $j=$ $1, . ., N-1$, and Supp $\psi_{N} \subset\{g=1\}$. Let us write

$$
\begin{aligned}
& \left(\psi\left(\hat{P}^{A D}\right)-\psi(\hat{P})\right) \psi_{1}\left(\hat{P}^{A D}\right) \hat{\Pi}_{g} \\
& =\left(\psi\left(\hat{P}^{A D}\right)-\psi(\hat{P})\right) \hat{\Pi}_{g} \psi_{1}\left(\hat{P}^{A D}\right) \hat{\Pi}_{g} \psi_{2}\left(\hat{P}^{A D}\right) \hat{\Pi}_{g} \ldots \hat{\Pi}_{g} \psi_{N}\left(\hat{P}^{A D}\right) \hat{\Pi}_{g} \\
& =\left(\psi\left(\hat{P}^{A D}\right)-\psi(\hat{P})\right) \hat{\Pi}_{g}\left(\psi_{1}\left(\hat{P}^{A D}\right)-\psi_{1}(\hat{P})\right) \hat{\Pi}_{g} \psi_{2}\left(\hat{P}^{A D}\right) \hat{\Pi}_{g} \ldots \hat{\Pi}_{g} \psi_{N}\left(\hat{P}^{A D}\right) \hat{\Pi}_{g} \\
& +\left(\psi\left(\hat{P}^{A D}\right)-\psi(\hat{P})\right) \hat{\Pi}_{g} \psi_{1}(\hat{P}) \hat{\Pi}_{g} \psi_{2}\left(\hat{P}^{A D}\right) \hat{\Pi}_{g} \ldots \hat{\Pi}_{g}\left(\psi_{N}\left(\hat{P}^{A D}\right) \hat{\Pi}_{g}\right.
\end{aligned}
$$

Applying (5.7) to the the pair $\left(\psi, \psi_{1}\right)$ we obtain

$$
\begin{aligned}
& \left\langle x_{2}-x_{1}\right\rangle^{s}\left(\psi\left(\hat{P}^{A D}\right)-\psi(\hat{P})\right) \psi_{1}\left(\hat{P}^{A D}\right) \hat{\Pi}_{g}\left\langle x_{2}-x_{1}\right\rangle^{s^{\prime}} \\
& =\left(\psi\left(\hat{P}^{A D}\right)-\psi(\hat{P})\right) \hat{\Pi}_{g}\left(\psi_{1}\left(\hat{P}^{A D}\right)-\psi_{1}(\hat{P})\right) \hat{\Pi}_{g} \psi_{2}\left(\hat{P}^{A D}\right) \hat{\Pi}_{g} \ldots \hat{\Pi}_{g} \psi_{N}\left(\hat{P}^{A D}\right) \hat{\Pi}_{g} \\
& +\mathcal{O}\left(h^{\infty}\right)
\end{aligned}
$$

Iterating this procedure and applying $(5.7)$ to the the pair $\left(\psi_{j}, \psi_{j+1}\right)$, one easily obtains

$$
\begin{aligned}
& \left\langle x_{2}-x_{1}\right\rangle^{s}\left(\psi\left(\hat{P}^{A D}\right)-\psi(\hat{P})\right) \psi_{1}\left(\hat{P}^{A D}\right) \hat{\Pi}_{g}\left\langle x_{2}-x_{1}\right\rangle^{s} \\
& =\left\langle x_{2}-x_{1}\right\rangle^{s}\left(\psi\left(\hat{P}^{A D}\right)-\psi(\hat{P})\right) \hat{\Pi}_{g}\left(\psi_{1}\left(\hat{P}^{A D}\right)-\psi_{1}(\hat{P})\right) \hat{\Pi}_{g} \ldots \ldots . . \\
& \quad \ldots \ldots . \hat{\Pi}_{g}\left(\psi_{N}\left(\hat{P}^{A D}\right)-\psi_{N}(\hat{P})\right) \hat{\Pi}_{g}\left\langle x_{2}-x_{1}\right\rangle^{s^{\prime}}+\mathcal{O}\left(h^{\infty}\right)
\end{aligned}
$$


Since it is easy to check (see also Lemma 4.3 in [KMW1]) that, for any $j=$ $1, . ., N$,

$$
\begin{aligned}
& \left\langle x_{2}-x_{1}\right\rangle^{s}\left(\psi\left(\hat{P}^{A D}\right)-\psi(\hat{P})\right) \hat{\Pi}_{g}\left\langle x_{2}-x_{1}\right\rangle^{s^{\prime}}=\mathcal{O}(h) \\
& \left\langle x_{2}-x_{1}\right\rangle^{s}\left(\psi_{j}\left(\hat{P}^{A D}\right)-\psi_{j}(\hat{P})\right) \hat{\Pi}_{g}\left\langle x_{2}-x_{1}\right\rangle^{s^{\prime}}=\mathcal{O}(h),
\end{aligned}
$$

we can conclude that the first term on the right hand side of (5.9) is of order $\mathcal{O}\left(h^{N+1}\right)$ and the Lemma follows.

Let us prove now our main Theorem:

Theorem 5.4. Under assumptions $(H 1),(H 2)$ and $(H 3)$, if $\mu_{0} \in \mathbb{R}$, satisfy

$$
\mu_{0}<\inf _{x} \inf \operatorname{Sp}\left(\tilde{Q}\left(x_{2}-x_{1}\right) \backslash \lambda_{1}\left(x_{2}-x_{1}\right)\right)
$$

then, there exists $\varepsilon>0$ such that, for $\chi \in C_{0}^{\infty}\left(\mu_{0}-\varepsilon, \mu_{0}+\varepsilon\right)$, Supp $\chi \subset\{g=1\}$, we have

$$
\left\|\left(\Omega_{ \pm}^{N A D}-1\right) \chi\left(\hat{P}^{A D}\right)\right\|=\mathcal{O}\left(h^{\infty}\right)
$$

and

$$
\left\|\left(\Omega_{ \pm}^{c}-\Omega_{ \pm}^{A D}\right) \chi\left(\hat{P}_{c}\right) \hat{E}\right\|=\mathcal{O}\left(h^{\infty}\right)
$$

as $h \rightarrow 0$.

Proof: Let us choose $\varepsilon>0$ such that $\left.\mu_{0}+\varepsilon<\inf \operatorname{Sp}\left(\hat{\Pi}_{g}^{\perp} \hat{P} \hat{\Pi}_{g}^{\perp}\right)\right)$ and such that the resolvent estimates of Proposition 5.1 hold. Let take $\chi, \phi \in C_{0}^{\infty}\left(\mu_{0}-\varepsilon, \mu_{0}+\varepsilon\right)$, Supp $\phi \subset\{g=1\}$, Supp $\chi \subset\{\phi=1\}$. We have

$$
\begin{aligned}
\left(\Omega_{+}^{N A D}-1\right) \chi\left(\hat{P}^{A D}\right) \hat{\Pi}_{g} & =\frac{i}{h} \int_{0}^{+\infty} e^{i t \hat{P} / h} V e^{-i t \hat{P}^{A D} / h} \chi\left(\hat{P}^{A D}\right) d t \\
& +(\phi(\hat{P})-1) \chi\left(\hat{P}^{A D}\right) \hat{\Pi}_{g}
\end{aligned}
$$

where

$$
V=\phi(\hat{P})\left(\hat{P}-\hat{\Pi}_{g} \hat{P} \hat{\Pi}_{g}\right) \hat{\Pi}_{g}=\phi(\hat{P})\left[\hat{P}, \hat{\Pi}_{g}\right] \hat{\Pi}_{g}
$$

Hence, by (5.5), (5.6) and (3.34)

$$
\begin{aligned}
& \left|\left\langle\int_{0}^{+\infty} e^{i t \hat{P} / h} V e^{-i t \hat{P}^{A D} / h} \chi\left(\hat{P}^{A D}\right) u d t, v\right\rangle\right| \\
& \leq\left\|\left\langle x_{2}-x_{1}\right\rangle^{\delta^{\prime} / 2} V\left\langle x_{2}-x_{1}\right\rangle^{\delta^{\prime} / 2}\right\|\|u\|\|v\| \leq C h^{N+1}\|u\|\|v\|
\end{aligned}
$$

for any $N \in \mathbb{N}$, for all $u, v \in L^{2}$, if $1<\delta^{\prime}<\delta$. Moreover, by Lemma 5.3

$$
(\phi(\hat{P})-1) \chi\left(\hat{P}^{A D}\right)=\left(\phi\left(\hat{P}^{A D}\right)-1\right) \chi\left(\hat{P}^{A D}\right)+\mathcal{O}\left(h^{\infty}\right) .
$$

Using (5.12), (5.13) and (5.14) we obtain (5.10). Moreover, writing

$$
\left(\Omega_{ \pm}^{c}-\Omega_{ \pm}^{A D}\right) \chi\left(\hat{P}_{c}\right) \hat{E}=\Omega_{ \pm}^{N A D} \phi\left(\hat{P}^{A D}\right) \Omega_{ \pm}^{A D} \chi\left(\hat{P}_{c}\right) \hat{E}=\Omega_{ \pm}^{A D} \chi\left(\hat{P}_{c}\right) \hat{E}+\mathcal{O}\left(h^{\infty}\right),
$$

we get (5.11). 


\section{References}

[Ba] A. BAlazARD-Konlein, Calcul fonctionel pour des opérateurs $h$ admissibles à symbole opérateurs et applications, Thése de Doctorat, Université de Nantes (1985).

[BoOp] M. Born, R. Oppenheimer, Zur Quantentheorie der Molekeln, Ann. Phys. 84, (1927), 457 .

[BrNo] R. Brummelhuis, J. Nourrigat, Scattering amplitude for Dirac operators, Comm. Partial Differential Equations 24 no. 1-2, (1999), 377-394.

[CDS] J.-M. Combes, P. Duclos, R. SeIler, The Born-Oppenheimer approximation, in: Rigorous atomic and molecular physics, G. Velo and A. Wightman (Eds.), Plenum Press New-York (1981), 185-212.

[CoSe] J.-M. Combes, R. SEILER, Regularity and asymptotic properties of the discrete spectrum of electronic Hamiltonians, Int. J. Quant. Chem. XIV (1978), 213-229.

[DiSj] M.Dimassi, J.SJÖSTRAnd , Spectral asymptotics in the semi-classical limit, London Mathematical Society Lecture Note Series, 268., Cambridge University Press, Cambridge, 1999.

[Ha1] G. Hagedorn, Semiclassical quantum mechanics IV, Ann. Inst. H. Poincaré 42 (1985), 363-374.

[Ha2] G. HagedoRn, High order corrections to the time-independent BornOppenheimer approximation I: Smooth potentials, Ann. Inst. H. Poincaré 47 (1987), 1-16.

[Ha3] G. HAGEDORN, High order corrections to the time-independent BornOppenheimer approximation II: Diatomic Coulomb systems, Comm. Math. Phys. 116 (1988), 23-44.

[Ha4] G. Hagedorn, A time-dependent Born-Oppenheimer approximation, Comm. Math. Phys. 77 (1980), 1-19.

[Ha5] G. HaGedoRn, High order corrections to the time-dependent BornOppenheimer approximation I: Smooth potentials, Ann. Math. 124, 571-590 (1986). Erratum. Ann. Math, 126 (1987), 219.

[Ha6] G. HaGedoRn, High order corrections to the time-dependent BornOppenheimer approximation II: Coulomb systtems, Comm. Math. Phys. 116 (1988), 23-44.

[HaJo] G. Hagedorn, A. Joye, A Time-Dependent Born-Oppenheimer Approximation with Exponentially Small Error Estimates, Comm. Math. Phys. 223, no. 3 (2001), 583-626. 
[HeSj1] B. Helffere, J. SJöstrand, Multiple wells in the semiclassical limit I, Comm. Part. Diff. Eq. 9, no.4 (1984), 337-408.

[Je1] T. Jecko, Sections efficaces totales dune molecule diatomique dans lapproximation de Born-Oppenheimer, Thése de Doctorat, Universit de Nantes (1996).

[Je2] T. Jecko, Classical limit of elastic scattering operator of a diatomic molecule in the Born-Oppenheimer approximation., Ann. Inst. H. Poincar, 69, (1998), 83-131.

[Je3] T. JEcko, Estimations de la rsolvante pour une molcule diatomique dans l'approximation de Born-Oppenheimer. , Comm. Math. Phys., 195, (1998), 585-612.

[Je4] T. Jecko, Approximation de Born-Oppenheimer de sections efficaces totales diatomiques, Asymt. Anal., 24,(2000), 1-35.

[JMP] A. Jensen, E.Mourre, P.Perry Multiple commutator estimates and resolvent smoothness in quantum scattering theory Ann. Inst. Henri Poincar, Phys. Thor. 41 (1984), 207-225.

[Ka] T. Kato, Perturbation Theory for Linear Operators, 2nd ed. Classics in Mathematics, Springer-Verlag, Berlin (1980).

[KMW1] M. Klein, A. Martinez, X.P. Wang, On the Born-Oppenheimer Approximation of Wave Operators in Molecular Scattering Theory, Commun. Math. Phys. 152, (1993), 73-95.

[KMW2] M. Klein, A. Martinez, X.P. Wang, On the Born-Oppenheimer Approximation of Diatomic wave operators II. Singular potentials, Journal Math. Phys. 38 no.3, (1997), 1373-1396.

[KMSW] M. Klein, A. Martinez, R. Seiler, X.P. Wang, On the BornOppenheimer Expansion for Polyatomic Molecules, Commun. Math. Phys. 143, no.3 (1992), 607-639.

[Ma1] A. Martinez, Développement asymptotiques et efffet tunnel dans l'approximation de Born-Oppenheimer, Ann. Inst. H. Poincaré 49 (1989), 239-257.

[Ma2] A. Martinez, An Introduction to Semiclassical and Microlocal Analysis, Universitext. Springer-Verlag, New York, 2002.

[MaSo1] A. Martinez, V. Sordoni, A general reduction scheme for the timedependent Born-Oppenheimer approximation, C.R. Acad. Sci. Paris, Ser. I 334 (2002), 185-188.

[MaSo2] A.Martinez, V.Sordoni, Twisted pseudodifferential calculus and application to the quantum evolution of molecules, Preprint (2006) (to appear in "Memoirs of the American Mathematical Society" (2007)). 
[Mo] E. MourRe, Absence of singular continuous spectrum for certain selfadjoint operators, Comm. Math. Phys. 78 (1981), 391-408.

[Ne1] G. Nenciu Linear Adiabatic Theory, Exponential Estimates, Commun. Math. Phys. 152 (1993), 479-496.

[Ne2] G. NeNCIU On asymptotic perturbation theory for quantum mechanics: almost invariant subspaces and gauge invariant magnetic perturbation theory J. Math. Phys. 43 (2002), 1273-1298.

[NeSo] G. Nenciu, V. Sordoni, Semiclassical limit for multistate KleinGordon systems: almost invariant subspaces and scattering theory, J. Math. Phys.,Vol. 45 (2004), 3676-3696.

[PST] G. Panati, H. Spohn, S. Teufel, Space-adiabatic perturbation theory, Adv. Theor. Math. Phys. 7 (2003).

[Ra] A. RAPHAELIAN, Ion-atom scattering within a Born-Oppenheimer framework, Dissertation Technische Universität Berlin (1986).

[Ro1] D. Robert, Autour de l'Approximation Semi-Classique, Birkhäuser (1987).

[Sj2] J. SJÖstrand, Projecteurs adiabatique du point de vue pseudodifferéntiel, C. R. Acad. Sci. Paris 317, Série I (1993), 217-220.

[So] V. SoRdoni, Reduction scheme for semiclassical operator-valued Schrödinger type equation and application to scattering, Comm. Partial Differential Equations 28 , no. 7-8(2003),1221-1236.

[SpTe] H. Spohn, S. Teufel, Adiabatic decoupling and time-dependent BornOppenheimer theory, Comm. Math. Phys. 224, no. 1, (2001) 113-132.

[Te] S. Teufel, Adiabatic Perturbation Theory in Quantum Dynamics, Lecture Notes in Math. 1821, Springer-Verlag, Berlin, Heidelberg, New York (2003),

[Wa] X.P.WANG Time-decay of scattering solutions and classical trajectories, Ann. IHP 47 (1987), 25-37, 